\title{
1 Phosphonate production by marine microbes: exploring new sources and \\ 2 potential function
}

Marianne Acker ${ }^{1,2, \dagger}$, Shane L. Hogle ${ }^{3,4 \dagger}$, Paul M. Berube ${ }^{3}$, Thomas Hackl ${ }^{3}$, Ramunas Stepanauskas $^{6}$, Sallie W. Chisholm ${ }^{3,5}$ and Daniel J. Repeta ${ }^{2, *}$

${ }^{1}$ MIT-WHOI Joint Program in Oceanography/Applied Ocean Science and Engineering, Woods Hole, Massachusetts, USA

${ }^{2}$ Department of Chemistry and Geochemistry, Woods Hole Oceanographic Institution, Woods Hole, Massachusetts 02543, USA

${ }^{3}$ Department of Civil and Environmental Engineering, Massachusetts Institute of Technology, Cambridge, MA 02139, USA

${ }^{4}$ Department of Biology, University of Turku, Turku 20500, Finland

${ }^{5}$ Department of Biology, Massachusetts Institute of Technology, Cambridge MA 02139, USA

${ }^{6}$ Bigelow Laboratory for Ocean Sciences, East Boothbay, Maine, 04544, USA

† These authors contributed equally to this work

Corresponding author : Correspondence and requests should be addressed to DJR (drepeta@whoi.edu) 


\section{Abstract}

Phosphonates, organic compounds with a C-P bond, constitute $20-25 \%$ of phosphorus in high molecular weight dissolved organic matter and are a significant phosphorus source for marine microbes. However, little is known about phosphonate sources, biological function, or biogeochemical cycling. Here, we determine the biogeographic distribution and prevalence of phosphonate biosynthesis potential using thousands of genomes and metagenomes from the upper 250 meters of the global ocean. Potential phosphonate producers are taxonomically diverse, occur in widely distributed and abundant marine lineages (including SAR11 and Prochlorococcus) and their abundance increases with depth. Within those lineages, phosphonate biosynthesis and catabolism pathways are mutually exclusive, indicating functional niche partitioning of organic phosphorus cycling in the marine microbiome. Surprisingly, one strain of Prochlorococcus (SB) can allocate more than $40 \%$ of its cellular P-quota towards phosphonate production. Chemical analyses and genomic evidence suggest that phosphonates in this strain are incorporated into surface layer glycoproteins that may act to reduce mortality from grazing or viral infection. Although phosphonate production is a low-frequency trait in Prochlorococcus populations ( $5 \%$ of genomes), experimentally derived production rates suggest that Prochlorococcus could produce a significant fraction of the total phosphonate in the oligotrophic surface ocean. These results underscore the global biogeochemical impact of even relatively rare functional traits in abundant groups like Prochlorococcus and SAR11.

\section{Introduction}

In nutrient-impoverished mid-ocean gyres, microbial demand for phosphorus $(P)$ is often so high that concentrations of inorganic phosphate are drawn down to sub nanomolar levels. Under these conditions, up to half of microbial $\mathrm{P}$ demand is met through the uptake and metabolism of P-containing dissolved organic matter ${ }^{1}$. Dissolved organic phosphorus (DOP) is a complex, 
poorly characterized mixture of high and low molecular weight (HMW and LMW) phosphate and

47 phosphonate esters. Phosphate esters are common in nucleic acids and lipids, and are synthesized by all marine microbes. The presence of phosphate esters in DOP is easily explained. In contrast, phosphonates, reduced P compounds with a stable, covalent C-P bond², are a poorly-understood component of marine DOP, but nevertheless constitute $20-25 \%$ of the $\mathrm{P}$ in HMWDOP${ }^{3}$.

All phosphonate production pathways are initially catalyzed through the same steps involving the enzymes PepM, Ppd, and Pdh² (Figure 1A). Despite these shared catalytic steps, the chemical diversity of phosphonates is extensive and includes small bioactive metabolites as well as macromolecules such as lipids, polysaccharides, and proteins. The functional roles and macromolecular forms that phosphonates take in marine microbes remain unknown. In contrast, phosphonate degradation potential in the marine environment is better understood ${ }^{4-6}$ (Figure 1B), and its distribution has been shown to be strongly shaped by phosphate availability ${ }^{7,8}$. Despite the high abundance of phosphonates in DOP, only two bacterioplankton (bacteria and archaea) species have been experimentally confirmed as phosphonate producers: Trichodesmium erythraeum, a nitrogen-fixing cyanobacterium ${ }^{9}$, and Nitrosopumilus maritimus from the Marine Group I (MGI) Thaumarchaeota ${ }^{10}$. Trichodesmium has a relatively restricted geographic range ${ }^{11}$ and global abundance, while MGI Thaumarcheota are abundant in the mesopelagic, but comparatively rare in sunlit surface waters ${ }^{12}$. Moreover, Candidatus Nitrosopelagicus brevis, the only characterized pelagic representative of MGI Thaumarcheota, lacks phosphonate biosynthesis genes and is significantly more abundant than Nitrosopulimus

67 in the open ocean ${ }^{13}$. It is unlikely that phosphonate production by Trichodesmium and

68 Thaumarcheota alone is enough to support the large and ubiquitous inventory of phosphonates observed in the sunlit upper ocean. Metagenomic surveys of PepM have estimated that between $8-16 \%$ of all marine microbes in the surface ocean may be capable of producing 
71 phosphonates ${ }^{10,14}$, but the taxonomic composition and ecological niches of phosphonate

72 producers remain unclear.

73 Much about the biology, ecology, and biogeochemistry of marine phosphonates remains to be

74 discovered. Recent vast expansions of marine genomic data ${ }^{15-18}$, as well as advances in

75 chemical analyses, makes it possible to systematically investigate the question of what

76 organisms are producing the enigmatic phosphonate pool in the oceans. Here we combine

77 laboratory studies and chemical analyses with comparative genomic and metagenomic

78 analyses to investigate the prevalence, taxonomic distribution, and potential function of

79 phosphonate biosynthesis in the surface ocean. We find that phosphonate biosynthesis genes

80 are found in a wide variety of marine microbes including the two most abundant groups in the

81 surface ocean: Prochlorococcus and SAR11. We experimentally demonstrate that a

82 Prochlorococcus strain produces phosphonates almost exclusively in the HMW protein fraction and, surprisingly, these phosphonoproteins account for over $40 \%$ of total cellular phosphorus.

\section{Main Text}

The taxonomic distribution of phosphonate producers and consumers

87 How widespread is the ability to produce and consume phosphonates among diverse marine microbes? Prior efforts to address this question have relied on mapping metagenomic reads to key marker genes such as the PepM found in phosphonate producers ${ }^{14}$ or the C-P lyase multienzymatic cluster and other catabolic pathway proteins found in phosphonate consumers ${ }^{4,6,7}$.

91 Here we expand on prior read recruitment methods and use a genome-resolved approach with

92 thousands of randomly sampled single-cell amplified genomes (SAGs) from the Global Ocean

93 Reference Genome (GORG-Tropics) dataset ${ }^{15}$. We found that the taxonomic richness of

94 phosphonate producers is higher than for consumers (Supplementary Note S1, Figure 2A).

95 However, the taxonomic evenness of producers was low, with over $60 \%$ of them assigned to 
SAR11 clades. In contrast, phosphonate consumers were more evenly distributed, but over $90 \%$ of SAGs came from just four taxonomic orders (Figure $2 \mathrm{~B}$ ). We found that the two most numerically abundant and cosmopolitan marine groups, SAR11 and Prochlorococcus are likely important phosphonate producers. SAR11 are small aquatic chemoheterotrophic Alphaproteobacteria estimated to constitute up to half of the total plankton cells in the surface $o_{\text {ocean }}{ }^{19}$, and Prochlorococcus are unicellular photosynthetic picocyanobacteria that numerically dominate the euphotic zone of subtropical and tropical oligotrophic areas ${ }^{20}$. Although the potential for phosphonate production is broadly taxonomically distributed, highly abundant pelagic groups like SAR11 and Prochlorococcus are likely to be the dominant producers across subtropical/tropical surface ocean.

We leveraged the GORG-Tropics reference database to quantitatively assess the proportion of cells in the surface ocean that are phosphonate consumers or producers. GORG-Tropics is buttressed by the use of a randomized cell selection strategy for generating SAGs - thus,

111 minimizing issues related to functional or taxonomic biases more common to targeted genome

112 sequencing. We estimate that, globally, $15 \%$ of all bacterioplankton in the upper 100 meters are 113 potential phosphonate producers in the GORG-tropics database. Compared to bacteria, 114 phosphonate producing archaea were rare in the euphotic zone ( $<3 \%$ of all GORG producers) 115 and phosphonate consuming archaea were absent (Figure 2A,B). Planktonic archaea 116 comprised only $14 \%$ of GORG-Tropics with $80 \%$ of archaea from Marine Group II and $20 \%$ from

117 Marine Group I. However, 37\% of Marine Group I SAGs contained phosphonate biosynthesis 118 genes. All phosphonate producing MGI archaea were isolated from below 100 meters and 119 included both Nitrosopumilus (20\%) and Nitrosopelagicus (80\%). Thus, archaea may be 120 important phosphonate producers at the base of the euphotic zone and mesopelagic, but within 
121 the sunlit ocean other bacterial producers dominate. Indeed, SAR11 (order Pelagibacterales) is

122 by far the most abundant producer constituting over $60 \%$ of all SAGs with phosphonate

123 biosynthesis potential (Figure $2 \mathrm{~A}$ ) and $18 \%$ of all SAR11 SAGs. Most phosphonate producers

124 are from the surface SAR11 clades 1a.3, Ila.1 and Ila.2, and the mesopelagic Ilb clade (Figure

$1252 \mathrm{~A}$, Supplementary Figure S1). The potential for methylphosphonate production is rare $(<1 \%$ of

126 genomes with MpnS) and is predominantly found in SAR11 SAGs (80\% of MpnS containing

127 SAGs are SAR11). Metagenome recruitment from earlier studies suggested Prochlorococcus

128 may account for $20 \%$ of phosphonate producers ${ }^{14}$. In GORG-Tropics we found that

129 Prochlorococcus constitutes 6\% of all phosphonate producers (Figure 2A) and 3\% of all SAGs.

130 Most Prochlorococcus phosphonate producers are from the surface high-light (HL) clades

131 (Supplementary Figure S1). The percentage of bacterioplankton that can produce (15\%) and consume (10\%) phosphonates is similar across the global surface ocean. On average there are three times more SAR11 phosphonate producers (18\%) than consumers $(5 \%)$, while for Prochlorococcus the trend is reversed - $6 \%$ producers versus $16 \%$ consumers (Supplementary Figure S2). SAR11 has been implicated as a major contributor to marine surface water methane supersaturation due to the metabolism of methylphosphonate ${ }^{21}$. Here our results imply that SAR11 has an even more important global role as a phosphonate producer.

We identified two major trends for producers and consumers in GORG-Tropics: 1) the abundance of phosphonate producers significantly increases with depth and is slightly biased

140 towards the Atlantic ocean (Figure 2C) and 2) consistent with past reports ${ }^{7}$ phosphonate 141 consumers are significantly more abundant in the Atlantic ocean (Figure 2D). The same trends 142 broadly held for SAR11 and Prochlorococcus consumers (Supplementary Figure S2). This 143 motivated us to look for other relationships between phosphonate producers and environmental 144 variables using a metagenomic dataset encompassing nearly 700 metagenomes from 145 bioGEOTRACES, Tara Oceans, and two long-term ocean time-series sites ${ }^{17,18,22}$. We 
determined the relative abundance of the PepM in the upper 250 meters of the global ocean using read recruitment to PepM and conserved marker genes (see methods). We estimate that the median proportion of phosphonate producers in the global ocean is $6 \%, 10 \%$, and $15 \%$ of Prochlorococcus, SAR11, and bacterioplankton genomes, respectively (Figure 3A), which agrees well with observations from GORG-Tropics. The discrepancy for SAR11 is likely explained by the low estimated sensitivity (60\%) for our method to SAR11 (Supplementary Note S5). Assuming we missed $40 \%$ of SAR11 PepM sequences, the corrected fraction of SAR11 is $17 \%$ which, again, agrees well with results from GORG. There was no statistically significant 154 difference in producer abundance between ocean basins (Supplementary Figure S3A), and no 155 significant time-averaged difference between the Hawaii Ocean Time-series (HOT) and the 156 Bermuda Atlantic Time Series (BATS) (Supplementary Figure S4), two long- running time series 157 representative of the N. Pacific and N. Atlantic subtropical gyres ${ }^{23,24}$. However, phosphonate producers have a significant seasonal dependence at the surface at BATS where producer abundance peaks during winter (November through the following March), which coincides with the peak of wind-driven deep mixing (Supplementary Figure S4, Supplementary Note S2).

161 Overall, the global median abundance for phosphonate producers is modest (15\%) and largely 162 stable across the global subtropical surface ocean.

Are there particular ocean features that may select for the ability of a microbe to produce 164 phosphonate? We used machine learning approaches ${ }^{25}$ (Supplementary Note S6) and 165 parametric regression ${ }^{26}$ (Supplementary Table S1) to identify biotic and chemical/physical 166 factors driving the distributions of Prochlorococcus, SAR11, and total bacterioplankton 167 phosphonate producers (Supplementary Figure S3). Generally, we find that the greatest amount 168 of phosphonate producer variation is explained by multiple depth-dependent biotic factors 169 (Figure 3B, Supplementary Figure S3 and S5, Supplementary Note S3). In surface samples 170 shallower than $100 \mathrm{~m}$, a median of $15 \%$ of bacterioplankton can produce phosphonates, while 
171 below $100 \mathrm{~m}$ that proportion steadily increases to nearly $30-40 \%$ at $200 \mathrm{~m}$. This $100 \mathrm{~m}$ break

172 coincides with the nutrient-driven "genomic transition zone" i.e. a zone where bacteria and

173 archaea tend to have larger genomes with higher GC content, and proteins with higher N/C

174 ratios $^{27}$, and implies that nutrient availability is an important factor setting the relative abundance

175 of phosphonate producers. Overall it appears that a modest proportion of highly abundant

176 oligotrophic "surface" bacterial clades including SAR11 la.3 and HL Prochlorococcus are

177 capable of producing phosphonates in the upper 50 to $100 \mathrm{~m}$ and contribute to an endemic

178 phosphonate pool there. Below $100 \mathrm{~m}$, the genetic potential for phosphonate production shifts to

179 other groups including SAR86, SAR11 Ilb, the OM1 clade, MGI Archaea and the ZD0417

180 clade $^{28}$. It may be that deep producers are sometimes uplifted into the upper $100 \mathrm{~m}$ of the

181 euphotic zone during seasonal mixing events or ephemeral upwelling events like cyclonic

182 mesoscale eddies ${ }^{29,30}$.

183 Our genomic and metagenomic results offer a number of predictions relevant to the

184 biogeochemistry of marine phosphonates. First, the vertical distribution of phosphonate

185 producers in the upper 250 meters is not even, with a distinct and rapid increase in producers

186 beginning at $100 \mathrm{~m}$ depth. This implies that bulk phosphonate production rates might be

187 similarly stratified, and that deeper phosphonate pools may be important for the total

188 phosphonate balance in the sunlit surface ocean. Second, unlike for phosphonate consumers ${ }^{7}$,

189 phosphate concentration does not appear to have a strong selective effect on the biogeography

190 of phosphonate producers in surface waters. However, this might be due to our statistical

191 approach not having a sufficient power to detect a true difference between ocean regions since

192 the mean proportions we compare are small with a relatively high variability. Further

193 investigation into ocean basin differences in phosphonate production potential is warranted.

194 Finally, the median proportion of bacteria and archaea that can produce phosphonates in the

195 upper 100 meters of the surface ocean appears to be relatively small (15\% of bacterioplankton 
cells). Since phosphonates appear to be relatively labile and rapidly cycled ${ }^{5}$, this implies that cellular production rates would need to be quite high to account for the large global inventory of phosphonates in marine dissolved organic matter.

\section{Phosphonate production in Prochlorococcus SB}

To better understand how much cellular $\mathrm{P}$ is allocated to phosphonate production and with which macromolecules they are associated, we sought to experimentally characterize phosphonate production by an abundant, widespread, and experimentally tractable marine microbe. We found only one cultured isolate that meets these criteria: Prochlorococcus SB. Since there is as yet no genetic system for knockout mutants in Prochlorococcus, we used Prochlorococcus MIT9301, a closely related strain lacking the phosphonate biosynthesis cluster, as a control. We analyzed cell pellets from each strain using ${ }^{31} \mathrm{P}$ nuclear magnetic resonance spectroscopy ( ${ }^{31} \mathrm{P}$-NMR). As expected, the ${ }^{31} \mathrm{P}$-NMR spectrum of MIT9301 cells shows that all $\mathrm{P}$ is allocated to phosphate and pyrophosphate esters (-12 to 12 ppm; Figure $4 \mathrm{~B}$ ). In contrast, ${ }^{31} \mathrm{P}$ NMR spectrum of Prochlorococcus SB cells displays strong signals between both -10 and 12 ppm from phosphate and pyrophosphate esters, and between 18 and 27 ppm from phosphonates $^{31}$ (Figure 4B). Integration of the phosphate and phosphonate ester regions of the NMR spectrum yields a cellular ratio of phosphonate to phosphate (Phn/Ph) of 0.72. (Figure 4B; Supplementary Table S3) which indicates that under nutrient replete conditions Prochlorococcus SB cells allocate $\sim 40 \%$ of their cellular P to phosphonate production.

\section{Phosphonates and $P$ storage}

The physiological and ecological roles of phosphonates are poorly understood. However, the presence of PepM among diverse genome-streamlined bacteria and the high relative abundance of cellular phosphonates in Prochlorococcus SB both suggest that phosphonates 
221 serve an important function for microbes inhabiting oligotrophic marine waters. One function

222 suggested for phosphonates is that they serve as an intra-cellular P-storage reservoir ${ }^{32}$. In

223 highly stratified oligotrophic waters, nutrient supply to phytoplankton is episodic; driven by

224 mixing events that bring nutrient-rich waters from below the surface into the euphotic zone. To

225 synchronize their need for nutrients with an episodic supply, some microbes take up excess $\mathrm{P}$

226 during periods of high nutrient concentrations and sequester them internally ${ }^{33,34}$. When nutrient

227 concentrations fall, internal P-stores are metabolized to release inorganic phosphate.

228 The Prochlorococcus SB genome lacks phosphonate degradation pathways including C-P

229 lyase, phosphonate hydrolytic pathways ${ }^{2}$, and $\mathrm{PhnYZ}$ an oxidative pathway recently discovered

230 in Prochlorococcus ${ }^{4}$. Recognizing that Prochlorococcus SB might use an uncharacterized

231 pathway to repurpose $\mathrm{P}$ from phosphonates, we tested the P-storage hypothesis in

232 Prochlorococcus SB by comparing phosphonate production in cultures grown under P-starved

233 and P-replete conditions (Figure 4A), expecting that if phosphonates were used for P-storage,

234 P-limitation would reduce the allocation of $\mathrm{P}$ to phosphonates. However, we found that

Prochlorococcus SB allocates significantly more $\mathrm{P}$ to phosphonates relative to phosphates upon

236 entering P-limited stationary phase growth $(\mathrm{Phn} / \mathrm{Ph}=2.4)$ than during $\mathrm{P}$-replete exponential

237 growth $(\mathrm{Phn} / \mathrm{Ph}=0.72$; Figure $4 \mathrm{~B})$. Cellular phosphorus to carbon $(\mathrm{C} / \mathrm{P})$ was relatively stable

238 across exponential and stationary phases (145 and 131 respectively) and changes in Phn/Ph

239 were driven by a decrease in phosphate ester content during P-starvation, with continued

240 production of phosphonates (Supplementary Figure S6, Supplementary Table S4). As

241 Prochlorococcus SB becomes increasingly P starved, the regulation of the cellular phosphate

242 and phosphonate pools becomes decoupled from exponential growth conditions. While

243 reallocation of $\mathrm{P}$ away from labile phosphates is one mechanism by which Prochlorococcus

244 adapts to P-starvation ${ }^{35}$, Prochlorococcus SB appears to be less able to regulate phosphonate

245 production or repurpose $\mathrm{P}$ in phosphonates towards other cellular functions. Both scenarios are 
inconsistent with phosphonates as a P-storage reservoir in Prochlorococcus SB, and P locked

247 into phosphonates is not internally recycled to sustain growth during periods of P-limitation.

248 This conclusion is reinforced by our comparative genomic analysis. Indeed, if phosphonates

249 were used by marine microbes for luxury P storage, we would expect both phosphonate

250 production and consumption pathways to coexist within the same genome. However, we found

251 that genomes with phosphonate biosynthetic potential almost never encode the machinery to

252 degrade and use phosphonates (Figure 5). In addition to biosynthesis genes, we searched for

253 all known phosphonate degradation pathways (Figure 1). In our analysis of the GORG-Tropics

254 and MARMICRODB ${ }^{36}$ genome datasets, we found that less than $1 \%$ of all genomes encoding at

255 least one marker gene implicated in phosphonate metabolism are both producers and

256 consumers. This mutual exclusivity occurs at the species/strain level - for example within HL

257 Prochlorococcus - which implies strong functional differentiation between even closely related

258 marine microbes. This suggests fine-scale niche partitioning between phosphonate producers

259 and consumers in the environment and may reflect functional incompatibility or

260 ecological/evolutionary tradeoffs between biosynthesis and catabolism. It also implies that if

261 phosphonates were a widespread storage strategy, most phosphonate producers would have

262 no way to reclaim $P$ from phosphonates during times of need.

\section{Macromolecular forms of phosphonates}

265 Past studies have linked the biochemical function of phosphonates to their macromolecular

266 form. For example, incorporation of phosphonates into membrane lipids or capsular

267 polysaccharides may protect cells against phospholipase activity or inhibit phage attachment ${ }^{37-}$

268 39. Therefore, we next asked what macromolecular form do phosphonates take in

269 Prochlorococcus SB? Phosphonate biosynthesis genes in most Prochlorococcus and SAR11

270 genomes are surrounded with glycosylating enzymes predicted to be involved in the 
271 biosynthesis of large extracellular polysaccharide structures, i.e. bacterial capsules

272 (Supplementary Figure S7, Supplementary Table S2). Many marine phytoplankton and bacteria

273 produce extracellular layers of polysaccharides to facilitate aggregation, for defense against

274 predation, and to manufacture biofilms ${ }^{40}$. Phosphonates have been shown to be constituents of

275 the large reservoir of dissolved polysaccharides that accumulate in the surface ocean ${ }^{5}$, and we

276 expected to find phosphonates in Prochlorococcus SB to be associated with polysaccharide

277 macromolecules. However, upon fractionation of Prochlorococcus organic matter into major

278 biochemical classes, we found that phosphonates were recovered in the protein fraction (Figure

279 4B) specifically within the methanol/acetone insoluble HMW protein fraction, which includes

280 membrane-associated proteins.

281 We reconcile these results by proposing that, in Prochlorococcus SB, phosphonates are

282 integrated into glycan polymer chains that are then post-translationally attached to HMW

283 proteins, likely membrane-anchored proteins. Indeed, one of the most common post-

284 translational modifications of bacterial proteins is O-linked glycosylation ${ }^{41}$, where glycan

polymers are covalently bound to serine or threonine side-chains of larger protein complexes.

286 The phosphonate gene clusters in Prochlorococcus SB, SAR11 strain HTCC7217, and SAR11

287 RS40 genomes are adjacent to glycan assembly enzymes and lipid carriers predicted to be

288 involved in the biosynthesis of capsules (Supplementary Figure S7). However, in some bacteria

289 the glycan building blocks for capsule biosynthesis are also routed towards post translational O-

290 linked glycosylation of membrane-bound lipoproteins ${ }^{42}$. Prochlorococcus SB genome contains

291 an O-oligosaccharyltransferase protein directly upstream of the PepM, Ppd, and Pdh cluster.

292 This protein family was first characterized in the attachment of glycans to liposaccharides, but

293 O-oligosaccharyltransferases have more recently been demonstrated to transfer preassembled

294 glycan chains onto protein substrates ${ }^{43,44}$. O-oligosaccharyltransferase genes are also present

295 near PepM in Pelagibacter strains HTCC7217 and RS40 (Supplementary Figure S7), and 15\%

296 of GORG-tropics SAGs contain PepM plus an O-oligosaccharyltransferase-like domain 
colocalized within the same $10 \mathrm{kbp}$ genome segment. This proportion increases to $50 \%$ if we relax the condition that the genes must occur on the same genomic contig. The genomic evidence implies that phosphonates could be a common moiety involved in the posttranslational O-linked glycosylation of proteins in the ocean.

\section{Functional roles of phosphonylated glycoproteins}

303 Why would an oligotrophic-adapted organism like Prochlorococcus use scarce $P$ to produce 304 large amounts of phosphonate-containing glycoproteins? Phosphonates are often incorporated 305 into cell-surface structures ${ }^{37,45}$ because they are highly resistant to hydrolysis and can inhibit the activity of some hydrolytic enzymes by mimicking carboxylic acids and phosphate esters ${ }^{46}$. For planktonic bacteria in the ocean, surface-expressed structures provide protection against enzymatic attack, UV-radiation, phages ${ }^{47}$, and protozoan grazers ${ }^{48}$. Modifying cell-surface structures with phosphonates may play a role in reducing vulnerability to grazers and phage, two major drivers of mortality for Prochlorococcus ${ }^{49}$. Given that surface expressed proteins are some of the most abundant proteins associated with the cell ${ }^{50}$, the large proportion of cellular $\mathrm{P}$ devoted to phosphonates in Prochlorococcus SB (Figure 4B) is also consistent with surface

313 protein modification.

314 Natural selection generally promotes high turnover and low population frequency of genes 315 involved in phage -and predator- interactions ${ }^{51}$ (Supplementary Note S4). Horizontal gene 316 exchange is a key mutational mechanism maintaining genes at low frequencies within microbial 317 populations ${ }^{51}$, and cell surface modification traits are highly enriched in horizontal gene 318 exchange networks ${ }^{52}$. Similarly, we find that patterns of phosphonate biosynthesis gene flow in 319 marine microbial communities do not follow a tree-like pattern and are better explained by 320 horizontal exchange and/or gene loss. Generally, there is a poor correlation between the 321 species tree, inferred from conserved marker genes, and the PepM tree. The topological 
distance between the species phylogeny and the PepM phylogeny approaches values for random simulated tree sets (Supplementary Table S5). In Prochlorococcus, nearly all

324 phosphonate biosynthesis gene cassettes are located within "genomic islands" (Supplementary

325 Figure S8), which contain the majority of laterally transferred genes in Prochlorococcus ${ }^{53,54 .}$

326 Indeed, there is also a transposase, an important molecular mechanism of lateral gene transfer

327 in bacteria ${ }^{55}$, four genes upstream of PepM in the Prochlorococcus SB genome. Taken together,

328 the low frequency of phosphonate producers in bacterial populations and the evidence for

329 horizontal transfer is consistent with a role for phosphonates within cell surface structures -

330 potentially as a defense against phage or grazers.

Above 50 meters we expect that chronic, long-term P scarcity drives the cost of phosphonate synthesis to outweigh its fitness benefit for most of the bacterioplankton. This is consistent with the numerical dominance of small, genome-streamlined cells, the low gene frequency of the phosphonate biosynthesis pathway, the low measured concentrations of particulate

337 phosphonates, and the low rates of phosphorus reduction relative to rates of community 338 phosphate uptake in the upper euphotic zone ${ }^{56,57}$. At the nutrient-driven genomic transition zone 339 and near the DCM, organic matter remineralization rates are relatively high and the 340 bacterioplankton community is in close proximity to episodic phosphate inputs from below.

341 Phosphate concentrations begin to rise, and the fitness benefit of phosphonate biosynthesis as 342 a defense against viral lysis and grazing begins to outweigh its cost (Figure 6), sustaining the 343 high frequency of phosphonate producers we found in this depth range. We expect that 344 phosphonates will be most abundant in particulate matter collected near the genomic transition 345 zone, and that higher rates of phosphonate production would lead to higher rates of 346 phosphonate degradation, consistent with the higher C-P lyase gene copy numbers and higher 
347

348

C-P lyase activity measured in microbes inhabiting the deep chlorophyll maximum ${ }^{7,58}$. If phosphonates are used to modify cell-surface glycoproteins as we suggest, then after cell death the labile protein component likely turns over quickly, but the inherently slow degradation of structural polysaccharides would lead to the accumulation of phosphonoglycans as high molecular weight dissolved organic matter ${ }^{59}$ explaining their abundance in marine DOP (Figure 6A). Therefore, a large fraction of dissolved organic $P$ in the ocean may be a direct result of grazer and viral defense mechanisms deployed by a small fraction of marine microbes that allocate a significant amount of their cellular P to phosphonate production.

\section{Acknowledgments}

We thank A. Coe, K. Dooley and C. Bliem for their invaluable help with Prochlorococcus cultivation; E. M. Grabowski, K. M. Bjorkman and D. M. Karl for their measurements of particulate carbon and phosphorus; C. G. Johnson for his assistance with the NMR. This work was supported in part by grants from the National Science Foundation (OCE-1153588, and DBI0424599 to S.W.C.; OCE-1335810 and OIA-1826734 to R.S. and OCE-1634080 to D.J.R), the Gordon and Betty Moore Foundation (\#6000 to D.J.R) and the Simons Foundation (Life Sciences Project Award IDs 337262, 647135, S.W.C.; 510023, R.S.; SCOPE Award ID 329108, S.W.C and D.J.R.). This paper is a contribution from the Simons Collaboration on Ocean Processes and Ecology (SCOPE).

\section{Authors contributions}

M. Acker grew the cultures, performed the macromolecular fractionation and protein extraction, acquired and interpreted the NMR spectra. S. L. Hogle performed all bioinformatics and biostatistics. T. Hackl assisted with genomic island prediction. P. M. Berube identified Prochlorococcus SB as a potential phosphonate producer and provided cellular biomass to D.J. 
372 Repeta for preliminary experiments. R. Stepanauskas provided early access to the GORG-

373 Tropics database. S. W. Chisholm supported the culture work by providing laboratory space,

374 training and advice. D. J. Repeta conceptualized the study, helped with data interpretation, and

375 provided general guidance on the execution of experiments. M. Acker, S. L. Hogle and D.J.

376 Repeta drafted the manuscript with assistance from all the co-authors.

377

378

379

380

381

382

383

384

385

386

387

388

389

390

391

392

\section{Methods}

Genomic data sources: We used two collections of genomes for the comparative genomics analysis: MARMICRODB ${ }^{36}$ (https://zenodo.org/record/3520509) and the Global Ocean Reference Genomes (GORG) Tropics dataset ${ }^{15}$. MARMICRODB contains over 18000 archaeal, bacterial, eukaryotic, and viral genomes from predominantly the marine environment, but also terrestrial and host-associated systems. GORG Tropics consists of approximately 13000 singlecell genomes sequenced from 28 samples across the tropical surface ocean. Roughly 6000 genomes from GORG Tropics come from a single sample (GORG BATS) from the Sargasso Sea. For quantitative analysis of the complete GORG dataset we randomly subsampled GORG BATS (Sample SWC-09) genomes to the median genomes per sample $(\mathrm{N}=241)$ from the remainder of GORG Tropics. We also used 195 surface and Deep Chlorophyll Maximum metagenomes from Tara Oceans project ${ }^{18}$ and 480 metagenomes from bioGEOTRACES, HOT, and BATS ${ }^{17}$. Genome and metagenome quality control and exclusion criteria were performed as described earlier ${ }^{36}$.

Homology searches: Phosphonate biosynthesis and catabolism proteins were identified by homology to a collection of hidden Markov models using HMMERv3.1b2 (http://hmmer.org/) and the trusted cutoffs of each individual model. PepM sequences were initially identified using PF13714 (PEP_mutase) and then aligned to the Tigrfam ${ }^{60}$ Hidden Markov Model (HMM) TIGR02320 using hmmalign. Authentic PepM sequences were identified by strict presence of 
conserved active site motif "EDK(X)5NS" or if they lacked at most two active site residues but were located adjacent (within 5000 nucleotides upstream/downstream) to a gene coding for Ppd (TIGR03297) or MpnS ${ }^{14,61-63}$. The PF13714 model identified many sequences lacking the PepM active site motif, and with significant similarity to the related isocitrate lyase superfamily PF00463. Sequences passing the PF13714 bitscore cutoffs, but lacking the "EDK(X)5NS" motif were classified as members of the isocitrate lyase superfamily. Sequences from authentic MpnS and the related HepDI/HepDII proteins were identified using custom HMMs built from alignments of curated sequences identified as containing essential catalytic residues ${ }^{64}$. These alignments were constructed using MAFFT v7.27365 in E-INS-i iterative refinement mode. The alignment was trimmed to peptide positions $317-792$ to isolate the core catalytic residues and used to construct HMMs. MpnS, HepDI, and HepDII sequences in MARMICRODB and GORGtropics were identified using the resulting HMMs (evalue $<0.05$ ), and the resulting sequences were aligned using MAFFT. The alignments were manually inspected to remove sequences lacking the essential catalytic residues and were iteratively realigned after poorly-aligned sequences were removed.

412 For phosphonate catabolism proteins, gene neighborhoods were inspected to confirm the 413 presence of multiple co-occurring genes from the $\mathrm{PhnYZ}^{4}, \mathrm{CP}$ lyase, 2-aminoethylphosphonate, 414 and phosphonoacetaldehyde phosphonatase catabolism pathways. Specifically, valid C-P lyase 415 clusters required at least five other genes from phnC, phnD, phnE, phnF, phnG, phnH, phnl, 416 phnJ, phnK, phnL, phnM, phnN, phnO, phnP to occur within uninterrupted (ie no scaffold/contig 417 breaks) 16000 nucleotide sliding windows. Valid phosphite clusters required two of ptxD, phnY, 418 and phnZ. Valid 2-aminoethylphosphonate clusters required co-occurring phnX and phnW or 419 phnW, phnA, and phnY. Valid phosphonoacetaldehyde phosphonatase clusters required phnZ, $420 \mathrm{hpnW}$, and hpnZ. All catabolic pathways other than C-P lyase required genes to co-occur within 421 uninterrupted 10000 nucleotide sliding windows. 
422 We also identified two sets of ten highly conserved, single-copy families from Prochlorococcus

423 and SAR11 lineages for use in normalization with metagenome profiling. These gene families

424 had the ten lowest $\mathrm{dN} / \mathrm{dS}$ ratios of the entire pangenome, that is the highest purifying/stabilizing

425 selection, for Prochlorococcus or SAR11, and should thus be highly specific and sensitive for

426 metagenomic quantification. Prochlorococcus and SAR11 pangenomes were characterized and

427 defined using PanX ${ }^{66}$. To profile metagenomes for total bacterioplankton, we used fetchMG

428 from the mOTUs2 tool ${ }^{67}$. Briefly, we identified ten COG families (COG0012, COG0016,

429 COG0018, COG0172, COG0215, COG0495, COG0525, COG0533, COG0541, COG0552) from

430 all bacterial and archaeal genomes in MARMICRODB. To reduce redundancy, we clustered

431 protein sequences from each COG at $90 \%$ sequence identity using MMseqs 2 v4e23d ${ }^{68}$ and

432 used the $90 \%$ clustered sequence representatives in metagenome search databases.

433 The HMM protein sequence profiles, results from these searches, and descriptions of the

434 sequence families are available from https://github.com/slhogle/phosphonates.

\section{Multiple sequence alignments, phylogenetic inference, and topological comparison:}

Authentic PepM sequences containing the "EDK(X)5NS" motif were aligned to TIGR02320

441 We used the GTDB-Tk v1.3.0 pipeline ${ }^{70}$ with default settings and the GTDB R05-RS95

442 database ${ }^{71}$ to identify conserved proteins (120 bacterial proteins/122 archaeal proteins) and

443 generate concatenated multi-protein alignments. We filtered alignment columns using the

444 bacterial and archaeal alignment masks from (http://gtdb.ecogenomic.org/downloads). We then

445 removed columns represented by fewer than $50 \%$ of all taxa and/or columns with no single

446 amino acid residue occurring at a frequency greater than $25 \%$. We further trimmed the 
447 alignments using trimAl with the automated -gappyout option to trim columns based on their gap

448 distribution. The multi-phylum genome phylogenies and the PepM phylogeny were inferred

449 using FastTree v2.1.1072 under the GAMMA model of rate heterogeneity and the WAG+

450 substitution mode ${ }^{73}$. Support values were determined using 100 non-parametric bootstrap

451 replicates. Both the PepM tree and genome tree were left unrooted. Phylogenies and

452 associated data were visualized using ggtree ${ }^{74}$. Detailed phylogenies of Prochlorococcus and

453 Pelagibacterales/SAR11 in the supplementary materials were constructed as described

454 earlier $^{36}$.

455 Comparisons between the topology of the PepM tree and the genome phylogeny were

456 performed using ETE3 v3.1.175. We pruned both the PepM and genome phylogenies so that

457 they contained the same number taxa and no duplication events in the PepM tree, which

458 resulted in 999 leaves for bacteria and 16 leaves for archaea. We then compared the topologies

459 of the PepM tree, the genome tree, and ten simulated random trees using the Robinson-Foulds

460 symmetric distance ${ }^{76}$ and the fraction of edge similarity. For Robinson-Foulds distances, edges

461 were treated as unpolarized splits rather than "true" clades because all trees were compared as

462 unrooted. The Robinson-Foulds distance simply counts the number of branch partitions (nodes)

463 that appear in one tree but not the other. Therefore, the maximum possible Robinson-Foulds

464 distances of an n-taxa unrooted tree is 2(n-3). To compute a normalized distance, we simply

465 divided the observed Robinson-Foulds distance by the maximum distance of the two-way

466 comparison between trees. Thus, the normalized Robinson-Foulds distance is a value from 0 to

467 1, which can be interpreted as the fraction of nodes/splits missing in the query tree compared

468 with the reference tree.

469 Gene enrichment analysis: We annotated the 16 Prochlorococcus and 22

470 SAR11/Pelagibacterales genomes containing verified PepM sequences with eggNOG 4.5.177

471 using eggNOG-Mapper v1.0.3-3-g3e22728 ${ }^{78}$. We then collected the resulting KEGG orthology 
472 annotations $^{79}$ from all genes and tested for enrichment of modules and pathways in the KEGG

473 hierarchy in genes within 10000 nucleotides upstream/downstream of the PepM sequence start

474 and stop coordinates respectively. Significant enrichment of KEGG categories were determined

475 using the hypergeometric test ${ }^{80}$ implemented in clusterProfiler v3.8 ${ }^{81}$. We excluded genomes

476 from the analysis that had contig/scaffold breaks within 10000 nucleotides

477 upstream/downstream of the PepM sequence.

478 Identification of PepM sequences within Prochlorococcus genomic islands: We predicted

479 genomic islands using Hidden Markov Models trained from conserved gene synteny patterns in

480 closed Prochlorococcus genomes. Briefly, we assume genomic islands to be contiguous

481 stretches of DNA enriched in flexible genes families (i.e. genes found in a subset of all

482 genomes). We then used previously described genomic islands in six Prochlorococcus

genomes ${ }^{54,82,83}$ to define core, flexible, and inconclusive gene "states" in four distinct hidden

markov models, each with two hidden states (island or non-island). We then used empirical

gene family frequencies as a proxy for the core/flexible/inconclusive state of each gene and the gene order on each scaffold as input to the Viterbi algorithm for predicting the hidden island

487 state of each gene in all other Prochlorococcus genomes.

Estimation of PepM prevalence in Prochlorococcus and SAR11 genomes: Because many

of the Prochlorococcus and SAR11 genomes analyzed here are incomplete (SAGs or MAGs),

490 we attempted to estimate the 'true' proportion of genomes with PepM while correcting for

491 genome incompleteness. Briefly, we used the estimated genome completeness from checkM

$492 \mathrm{v} 1.0 .11^{84}$, which is based on the presence of core marker genes, to estimate the number of

493 missing bases per taxonomic group (Prochlorococcus or SAR11) and then use this scale the

494 relative abundance of potential phosphonate producers per group. We estimated the corrected

495 prevalence of phosphonate producers as: 


$$
\left(\sum_{i=1}^{n} p / \sum_{i=1}^{n} g\right) \times \sum_{i=1}^{n}(g / c) \times \bar{p}^{-1} \times n^{-1}
$$

where for each clade, $p$ is the length of the phosphonate biosynthesis/degradation operon in base pairs, $g$ is the length of the genome assembly in base pairs, $c$ is the completeness estimate from CheckM, $\mathrm{p}$ is the average length of all phosphonate biosynthesis/degradation operons from each clade, and $n$ is the total number of assemblies from each clade.

502 Metagenome read classification: We created Diamond v0.9.22.123 ${ }^{85}$ search databases for 503 each of the three ten-gene marker gene sets identified for Prochlorococcus, SAR11, and all 504 bacterioplankton using reference genomes from MARMICRODB. We also created Diamond 505 databases for only Prochlorococcus PepM, only SAR11 PepM, and all bacterial and archaeal 506 PepM sequences identified with the "EDK(X)5NS" catalytic motif from MARMICRODB only. We identified the marker gene sets using a two-tiered search strategy using Diamond in default fast mode. We first searched the metagenomes against reduced marker sets clustered at $70 \%$ amino acid identity by MMseqs2. We pulled all reads with hits to these reduced marker sets, then searched the reads against the entire MARMICRODB, and finally retained read mappings

511 with a best scoring match to a MARMICRODB protein from the original marker family. We 512 searched metagenomic reads against the PepM databases using Diamond (mode --more513 sensitive) and score cutoffs of $55 \%$ amino acid identity and an E-value of 1 e- 5 . We determined 514 these cutoffs empirically to be those that produced the highest F Score (harmonic mean of 515 precision and recall) from mock metagenomes simulated from GORG-tropics (Supplementary 516 Note S5, Supplementary Figure S9). Since the E-value is a function of database size it is 517 important to note that the significance of this cutoff is specific to the reference databases here. 518 In the case of ambiguous alignments (i.e., identical best alignment scores to sequences from 
different taxonomic groups) we classified reads using a probability-weighted random sampling of all taxonomic groups matching the best hits. We derived the probability distributions for

521 random samplings from the taxonomic classification uniquely mapped PepM reads.

522 To normalize PepM reads across metagenomes we calculated the length normalized counts

523 (RPKM) of the 10 single copy marker genes from Prochlorococcus, SAR11, and all bacteria and 524 archaea in each metagenome as reads per kilobase marker and then estimated the number of 525 'genome equivalents' in each metagenome as the median of the 10 marker families. We also 526 calculated the length normalized abundance of PepM from Prochlorococcus, SAR11, and all

527 bacteria and archaea as reads per kilobase from each metagenome. We then divided the length

528 normalized abundance of PepM by the estimated number of genome equivalents for each

529 taxonomic group to estimate the fraction of genomes with phosphonate biosynthesis potential.

530 To ensure robust estimates of normalized abundance we excluded samples where the median marker gene coverage was less than 100X for all taxonomic groups.

532 Biotic and abiotic data associated with metagenomes: The biotic and abiotic variables used

533 in this study were obtained and preprocessed as described in detail here:

534 https://doi.org/10.5281/zenodo.3689249 and here: https://doi.org/10.5281/zenodo.3786232. We 535 obtained phosphate concentrations from the GEOTRACES Intermediate Data Product IDP2017 536 version $3^{22}$, specifically from sections $\mathrm{GA} 02^{86,87}, \mathrm{GA} 03, \mathrm{GA} 10^{88}$, and GP13. We obtained

537 dissolved phosphate concentrations from the Tara Oceans project ${ }^{89}$

538 (https://doi.pangaea.de/10.1594/PANGAEA.875579). Modeled climatological dissolved organic

539 phosphorus and other variables were obtained from the MIT Darwin model (v0.1_llc90,

540 http://darwinproject.mit.edu/) from the Simons Collaborative Marine Atlas project (CMAP)

541 https://simonscmap.com/ using pycmap v0.1.2 (https://doi.org/10.5281/zenodo.3561147).

542 Taxonomic profiles derived from metagenomic reads were generated as described earlier ${ }^{36}$

543 using the MARMICRODB database. As a first order estimate of Prochlorococcus and SAR11 
544

545

546

547

548

ecotype relative abundance we divided the number of reads mapping to each ecotype by the number of reads mapping in total the Prochlorococcus genus or the family Pelagibacterales for SAR11. Some proportion of reads mapping to highly conserved (core) regions can only be reliably classified at the genus or family level thus our ecotype relative abundance estimates using total read counts are underestimates.

Random Forest Regression: We trained one random forest model ( $\left.n_{\text {trees }}=1000\right)$ for each taxonomic group; Prochlorococcus, SAR11, and bacteria plus archaea. Each model was trained using up to 44 abiotic/biotic variables including trace metal and macronutrient data from GEOTRACES and Tara Oceans, modeled climatological means from the MIT Darwin model (http://darwinproject.mit.edu/), and ecotype relative abundances. For the outcome variable (normalized PepM relative abundance) we used a modified splitting rule for tree construction that maximized the log-likelihood of the beta distribution on the interval $[0,1]^{90}$. Although our relative abundance measure is not theoretically restricted to the unit interval (values greater than one could exist, e.g. if the PepM copies per genome greatly exceeded one), in practice PepM relative abundance was always bounded $[0,1]$ in our datasets. We performed random forest regression using nested ten-fold cross-validation to prevent data leakage to the validation phase ${ }^{91}$. We reserved $20 \%$ of the data for estimating final model performance. The remaining $80 \%$ of training data was split into 10 resampled partitions, each with analysis and assessment partitions, to tune and estimate the performance of preprocessing, supervised feature selection, hyperparameter tuning steps. We tuned hyperparameters (mtry, min.node.size), by maximizing the coefficient of determination from correlation (model $\mathrm{R}^{2}$ ) and minimizing the root mean square error (RMSE). We used CAR Scores ${ }^{92}$ for recursive feature elimination to retain only the top 50th percentile of informative variables. We included the feature elimination step to reduce the computation costs and runtime of the feature importance step (see below). Random forest regression was implemented with the package Ranger ${ }^{25}$ and the Beta Forest algorithm ${ }^{90}$. We 
569

570

571 572 importance level, i.e. random data.

573

574

575

576

577

578

579

580

581

582

583

584

585

586

587

588

589

590

591

592

593

determined predictor variable rankings on the final model from the cross validation step using the Boruta heuristic ${ }^{93}$. This step allowed us to identify all predictor variables that consistently performed better than chance and to compare the importance of each variable to a reference

\section{Beta-Binomial Regression and Generalized Additive Models: We used the R package} corncob $^{26}$ and modeled PepM relative abundance directly from PepM read counts and the median read counts to marker gene sets as "successes/total" which is appropriate for the betabinomial probability distribution. We used the log-odds link function for both relative abundance and the overdispersion parameter. For each taxonomic group (SAR11/Prochlorococcus/bacteria and archaea) we modeled only the top five most important biotic/abiotic variables identified in the Random Forest regression and variable importance steps. We did not include additional model terms because multiple collinearities between covariates and the additional model complexity prevented model convergence in most cases. We estimated the probability for each biotic/abiotic covariate being informative to the overall model by using bootstrapped likelihood ratio tests $(\mathrm{N}=1000)$. We estimated $95 \%$ confidence intervals for model coefficients and standard errors using 1000 random draws from the beta-binomial distribution.

We estimated seasonal effects in time-series metagenomes using Generalized Additive Mixed Models and Linear Mixed-Effect Models implemented through the mgcv v1.8-26 ${ }^{94} \mathrm{nlme}$ v3.1-148 libraries in R v3.6.2. To decompose any potential seasonal effects, we fit a cyclic spline term to a variable for the day of the year, which we use as a proxy for season, and we fit a global trend term to the cumulative time since sampling onset. We considered a seasonal effect present if the model term for "day of the year" was statistically significant $(p<0.05)$.

Prochlorococcus cultures under P-replete and P-deficient conditions: To investigate the linkage of PepM with phosphonate production, we grew two HLII strains of Prochlorococcus: Prochlorococcus SB, which has PepM, and the closely related Prochlororoccus MIT9301, which 
594 lacks the PepM gene sequence. Both strains were grown axenically, under constant light (30 $595 \mu \mathrm{mol}$ quanta $\mathrm{m}^{-2} \mathrm{~s}^{-1}$ ) in artificial seawater medium AMP1 prepared has described before ${ }^{95}$, but 596 using $3.75 \mu \mathrm{M}$ TAPS as a buffer instead of $1 \mathrm{mM}$ HEPES. After growing Prochlorococcus SB in 597 the regular medium i.e with a phosphate concentration of $50 \mu \mathrm{M}(\mathrm{N} / \mathrm{P}=16 / 1)$ to assess whether 598 or not this strain could produce phosphonate, we decreased the phosphate concentration to $5992.28 \mu \mathrm{M}(\mathrm{N} / \mathrm{P}=350 / 1)$. Although the phosphate concentration was lower, Prochlorococcus SB 600 cells were not P-limited in exponential phase growth. However, stationary phase was induced 601 by P-starvation, as demonstrated by the initiation of further growth after adding phosphate to the 602 culture (Figure 4A inset). For all experiments, biological duplicates were grown to ensure 603 reproducibility. Cultures axenicity was assessed by flowcytometry and by confirming a lack of 604 turbidity for at least 30 days after inoculation with three test broths: ProAC ${ }^{96}, \mathrm{MPTB}^{97}$ and 605 ProMM (Pro99 medium ${ }^{95}$ supplemented with $1 \times \mathrm{Va}$ vitamin $\mathrm{mix}^{98}$ and $0.05 \% \mathrm{w} / \mathrm{v}$ each of pyruvate, acetate, lactate and glycerol. ProMM is the $100 \%$ seawater based version of the 607 PLAG medium ${ }^{96}$. All the glassware and polycarbonate bottles $(1 \mathrm{~L}$ for the blank, $2 \mathrm{~L}$ and $20 \mathrm{~L}$ for 608 the cultures) were cleaned by soaking overnight in $2 \%$ detergent (micro), rinsed 6 times with 609 deionized water, soaked overnight in $1 \mathrm{M} \mathrm{HCl}$ and rinsed 6 times with ultra-high purity water.

Cell harvest and treatment: Prochlorococcus SB cultures grown in high N/P medium were

611 harvested twice: $\sim 6 \mathrm{~L}$ were harvested during exponential growth and the remainder $(14 \mathrm{~L})$

612 harvested two days after the onset of stationary phase growth. To ensure that stationary phase 613 cells were limited by phosphorus, $25 \mathrm{~mL}$ of culture was amended with phosphate. Fluorescence 614 increased in the phosphate-amended cultures, reaching levels regularly observed in

615 Prochlorococcus HLII cultures (Figure 4A inset). Cells were separated from the growth medium 616 by centrifugation $\left(15,970\right.$ rcf for 30 minutes at $\left.4^{\circ} \mathrm{C}\right)$ and the growth medium was saved for other 617 analyses. Cell pellets were transferred into $50 \mathrm{~mL}$ falcon tubes, suspended in Turk Island mix ${ }^{95}$ 618 to rinse the cells of external nutrients and centrifuged $\left(6,523 \mathrm{rcf}\right.$ for 15 minutes at $\left.15^{\circ} \mathrm{C}\right)$. This 
time, we discarded the supernatant and repeated the operation two more times. After, we flash

620 froze the cell pellets in liquid nitrogen and stored them at $-20^{\circ} \mathrm{C}$ until NMR analyses. We also

621 measured Prochlorococcus cell abundance by flow cytometry. Samples were prepared and

622 processed as previously described ${ }^{99,100}$ a d run on an Guava 12HT flow cytometer (Luminex

623 Corp., Austin , TX, USA). Cells were excited with a blue $488 \mathrm{~nm}$ laser analyzed for chlorophyll

624 fluorescence (692/40nm), SYBR Green I stained DNA fluorescence content (530/40nm), and

625 size (forward scatter). Samples were ran stained with 1x SYBR Green I (Invitrogen, Grand

626 Island, NY) and unstained then incubated for $60 \mathrm{~min}$ in the dark prior to running. All flow

627 cytometry files were analyzed using Guavacyte.

628 Nuclear Magnetic Resonance: NMR spectra were acquired at $25^{\circ} \mathrm{C}$ on a $400 \mathrm{MHz}$ Bruker

629 AVANCE DPX spectrometer using a 5mm inverse broadband probe and running TOPSPIN 1.3.

$630{ }^{31} \mathrm{P}$ shifts are reported relative to external $85 \%$ phosphoric acid at $0 \mathrm{ppm}$. For the proton-

631 decoupled ${ }^{31}$ P-NMR spectra, we used 'zgdc30' with WALTZ16 decoupling and sweep width of

63280 ppm, a 3 seconds relaxation delay, $100 \mathrm{~K}$ scans and $20 \mathrm{~Hz}$ line broadening. Prochlorococcus

633 SB and MIT9301 whole cells were packed into a 5mm BMS tube (Shigemi Inc.) with magnetic

634 susceptibility of the glass inserts matching $\mathrm{D}_{2} \mathrm{O}$.

$635{ }^{31} \mathrm{P}-\mathrm{NMR}$ spectra of Prochlorococcus SB protein fraction was acquired at $25^{\circ} \mathrm{C}$ on a $400 \mathrm{MHz}$

636 Bruker Ascend 400 equipped with a Sample CASE. The ${ }^{31} \mathrm{P}-\mathrm{NMR}$ spectra were acquired using

637 the program 'zgpg30' with a sweep width of $80 \mathrm{ppm}$, a relaxation delay of 2 seconds, a $15 \mathrm{~Hz}$

638 line broadening and for $13 \mathrm{~K}$ scans. ${ }^{31} \mathrm{P}$ chemical shifts are reported relative to external

639 phosphoric acid at 0 ppm.

640 Elemental composition of Prochlorococcus SB and MIT 9301: Elemental C/N/P ratios were

641 measured at the University of Hawai'i nutrient facility according to the protocols employed by the

642 Hawaii Ocean Time series program

643 (http://hahana.soest.hawaii.edu/hot/protocols/protocols.html\#). Briefly, cell pellets from 900 
$644 \mathrm{~mL}$ of culture were transferred to combusted glass vials, dried, and powdered. $\mathrm{C}$ and $\mathrm{N}$ were

645 measured on subsamples using a PE-2400 Carbon/Nitrogen analyzer calibrated with acetanilide

646 standards. Cellular $P$ was measured by the molybdenum blue method ${ }^{101,102}$ after first

647 combusting cell pellets at $450^{\circ} \mathrm{C}$ for $3 \mathrm{~h}$, and dissolving the residue in $10 \mathrm{~mL}$ of $0.5 \mathrm{M} \mathrm{HCl}$.

648 Separation of cellular macromolecular classes: To fractionate Prochlorococcus organic

649 matter into different classes of major biochemicals, we followed the protocols of Karl et al. ${ }^{103}$.

650 Cells from $3 \mathrm{~L}$ of culture were centrifuged and the isolated cell pellet extracted with $5 \mathrm{~mL}$ of cold

$6515 \%$ trichloroacetic acid (TCA) for $1 \mathrm{~h}$. The mixture was centrifuged (12,100 rcf for 30 minutes at

$6524^{\circ} \mathrm{C}$ ), the supernate decanted, and the TCA insoluble material washed twice with $5 \%$ cold TCA.

653 The TCA fractions were combined, evaporated to dryness. Lipids were recovered from the TCA

654 insoluble material by extraction (3x, room temperature, 20 minutes each) with $95 \%$ ethanol (5

$655 \mathrm{~mL})$. Residual ethanol was evaporated, and RNA within the dry TCA/95\% ethanol insoluble

656 material hydrolyzed at $37^{\circ} \mathrm{C}$ for 1 hour with $2.5 \mathrm{~mL}$ of $1 \mathrm{M} \mathrm{NaOH}$. The hydrolysis was quenched

657 by immersing the sample tube in an ice bath for 15 minutes, after which the sample was

658 acidified to $\mathrm{pH} 1$ by adding $2.5 \mathrm{~mL} 1 \mathrm{M} \mathrm{HCl}$ and $0.5 \mathrm{~mL}$ of $50 \%$ TCA. The mixture was allowed

659 to sit for 15 min to precipitate proteins and DNA, then centrifuged for 30 minutes at 12,100 rcf.

660 After collecting the supernatant (containing RNA), we rinsed and centrifuged the pellet $2 x$ with 5

$661 \mathrm{~mL}$ of $5 \%$ TCA and $2 x$ with $5 \mathrm{~mL}$ of $95 \%$ ethanol. To hydrolyze DNA, we added $5 \mathrm{~mL}$ of $5 \%$ TCA

662 to the insoluble material and immersed the tube in boiling water for 30 minutes. After

663 centrifuging the tube and collecting the DNA-containing supernatant, we rinsed and centrifuged

664 the pellet $2 x$ with $5 \mathrm{~mL}$ of ice-cold $5 \%$ TCA and $2 x$ with ice-cold $95 \%$ ethanol as before. Finally,

665 we extracted the protein from the remaining cell pellet with $5 \mathrm{~mL}$ of $1 \mathrm{M} \mathrm{NaOH}\left(37^{\circ} \mathrm{C}, 18 \mathrm{~h}\right)$. A

666 small amount of insoluble debris remained after the protein extraction. This was removed by

667 centrifugation (12,100 rcf; 30 minutes) and followed by syringe filtration of the supernatant. 
Protein extraction and precipitation: Peptides and denatured "soluble" proteins were fractionated from native HMW "insoluble" proteins using the protocol described by Hutchins et al. ${ }^{104}$. The cell pellet of $0.5 \mathrm{~L}$ of culture was lysed (15 minutes, RT) with $1 \mathrm{~mL}$ of $1 \% \mathrm{SDS}$

671 extraction buffer ( $1 \%$ SDS, $0.1 \mathrm{M} \mathrm{Tris} / \mathrm{HCl} \mathrm{pH} \mathrm{7.5,10} \mathrm{mM} \mathrm{EDTA)} \mathrm{then} \mathrm{heated} \mathrm{at} 95^{\circ} \mathrm{C}$ for 10

672 minutes. Samples were allowed to cool to RT and were then agitated at $350 \mathrm{rpm}$ for 1 hour. The

673 resulting suspension was centrifuged (20 minutes, 14,100 rcf), and the supernatant decanted.

674 After transferring the supernatant containing the proteins, we concentrated the proteins from the

675 supernate by membrane centrifugation using $5 \mathrm{~K}$ molecular weight cutoff Vivaspin units of $6 \mathrm{~mL}$

676 (Sartorius Stedim, Goettingen, Germany). The retentate ( 300 $\mu \mathrm{L})$ was recovered, and $1 \mathrm{~mL}$ of cold $50 / 50$ methanol/acetone solution (acidified with $\mathrm{HCl}$ to a final concentration of $0.5 \mathrm{mM}$ ) added. After sitting for 3 days at $-20^{\circ} \mathrm{C}$, insoluble proteins were pelleted by centrifuge $(14,100$ rcf for 30 minutes at $4^{\circ} \mathrm{C}$ ) and the supernatant decanted.

Dataset/code availability: The datasets and computer code supporting the findings in this study are available from: https://github.com/slhogle/phosphonates.

682 The entire MARMICRODB dataset including a comprehensive description, raw protein fasta 683 files, Kaiju v1.6.0105 formatted databases, scripts and instructions for how to use the resource is 684 available from https://doi.org/10.5281/zenodo.3520509.

685 GEOTRACES chemical data was processed and matched to metagenome samples using 686 code/methods available from https://doi.org/10.5281/zenodo.3689249.

687 Tara Oceans chemical and hydrographic data was processed and matched to metagenome 688 samples using code/methods available from https://doi.org/10.5281/zenodo.3786232.

689 The list of Prochlorococcus core PFAM and TIGRFAM families, a compiled HMMERv3 hidden 690 Markov model database, and a CheckM formatted marker list file is available from $691 \quad$ https://doi.org/10.5281/zenodo.3719132. 
A) Phosphonate Production

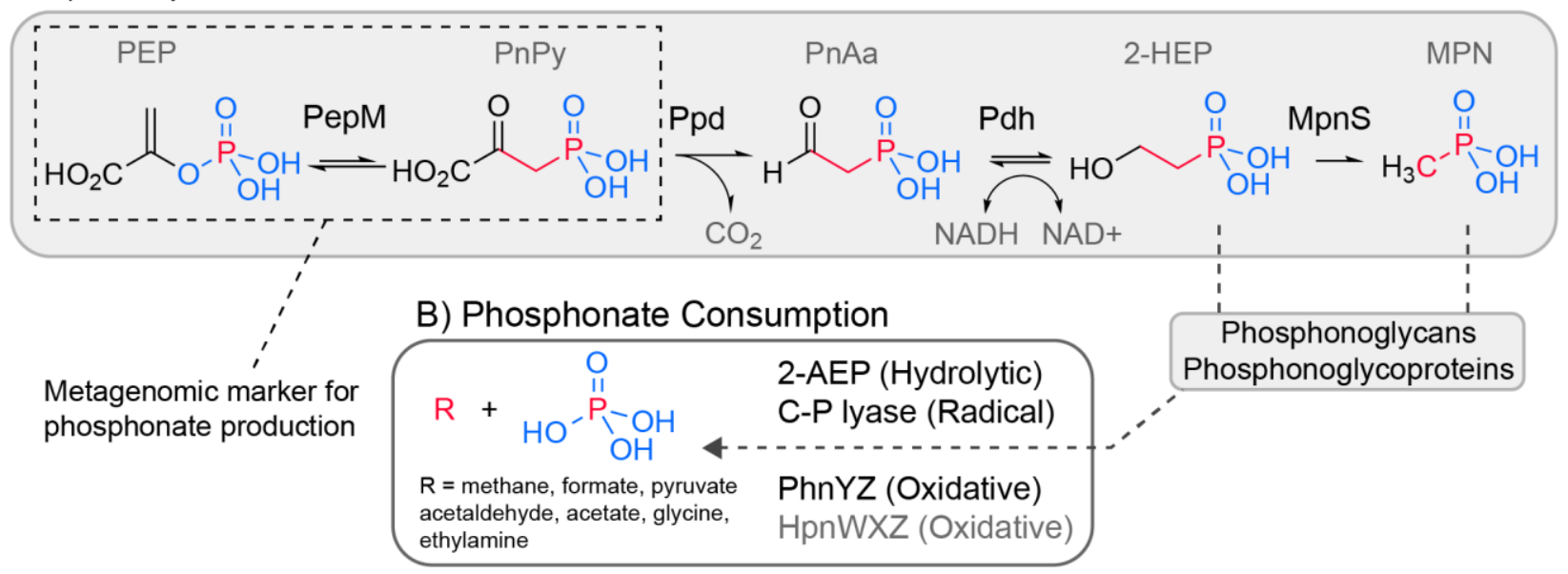
A) phosphonopyruvate (PnPy) via phosphoenolpyruvate mutase (PepM), (2) decarbo of PnAa to 2-hydroxyethylphosphonic acid (2-HEP) via phosphonoacetaldehyde dehydrogenase (Pdh). 2HEP can be further transformed by methylphosphonate synthase (MpnS), to produce methylphosphonate (MPn), a substrate for aerobic marine methane production ${ }^{5}$. Phosphonates in the marine environment are

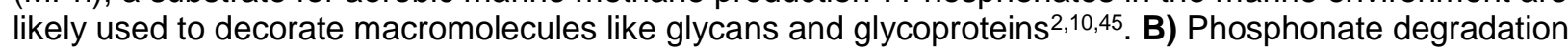
via cleavage of the C-P bond proceeds through at least three mechanisms: hydrolytic (2AEP, named for the representative 2-aminoethylphosphate degradation pathway via PhnWX), radical (C-P lyase), and oxidative (PhnYZ). The proposed HpnWXZ pathway is likely oxidative proceeding via the oxidative deamination of an aminophosphonate similar in structure to 2-aminoethylphosphate ${ }^{106}$. We find the HpnWXZ pathway to be rare in marine genomes. 
A Phosphonate Producers

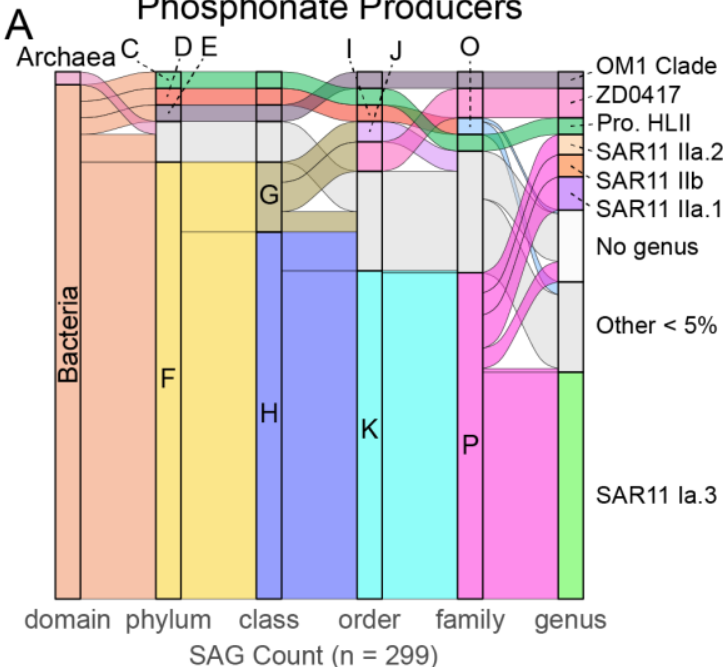

Phylum

$\mathrm{C}=$ Cyanobacteria

$\mathrm{D}=$ Bacteroidota

$\mathrm{E}=$ Actinobacteria

$\mathrm{F}=$ Proteobacteria

C

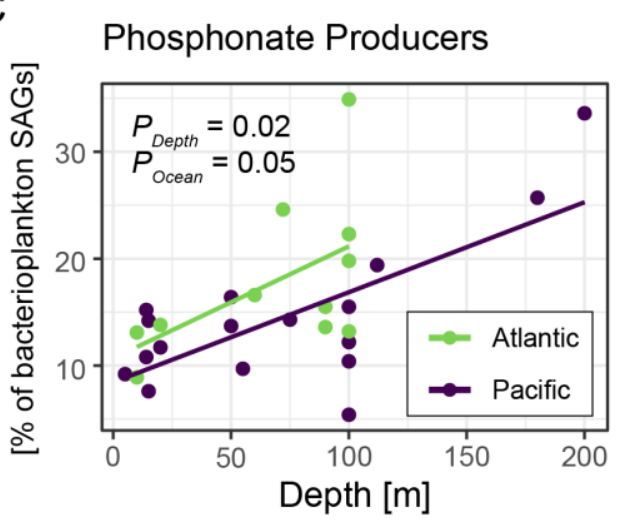

B Phosphonate Consumers

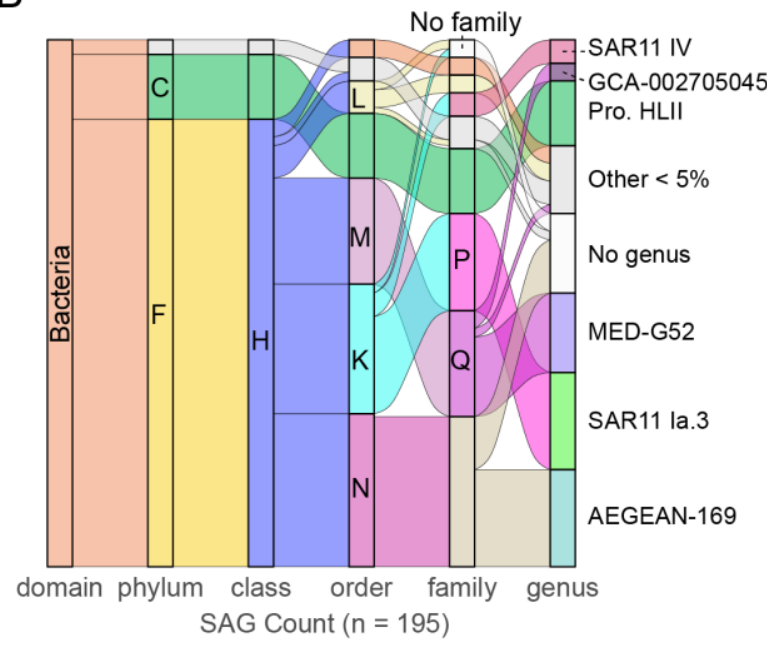

Order

I = Flavobacteriales $M=$ Rhodobacterales

$\mathrm{J}=\mathrm{SAR} 86 \quad \mathrm{~N}=\mathrm{HIMB} 59$

Family

G = Gammaproteobacteria $\mathrm{H}=$ Alphaproteobacteria

$\mathrm{K}=$ Pelagibacterales $\mathrm{L}=\mathrm{SAR} 116$

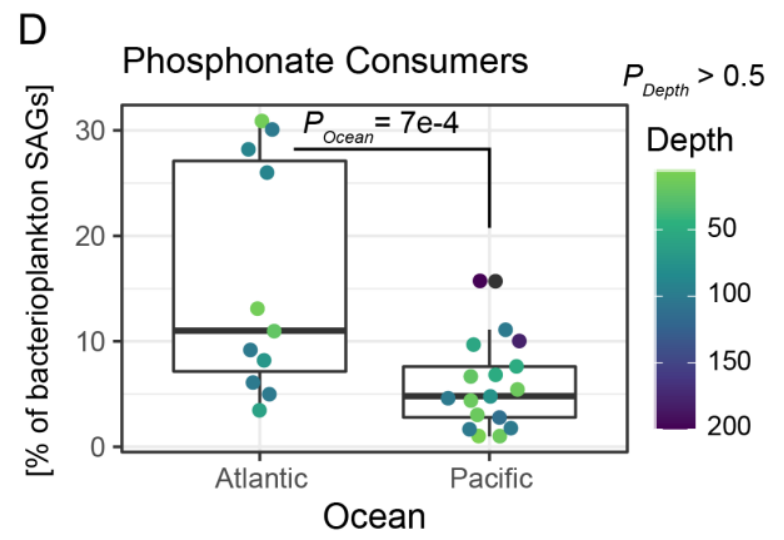

Figure 2: Genomic potential for phosphonate production and consumption in nearly 13000 single-cell genomes from the tropical ocean

Flow diagrams representing taxonomic hierarchy of $\mathbf{A}$ ) phosphonate producers and $\mathbf{B}$ ) consumers from the GORG-Tropics database ${ }^{15}$. Flows of the same color spanning multiple taxonomic ranks indicate that the higher ranks are entirely subsumed by the lowest rank. For example, all cyanobacterial genomes were from the Prochlorococcus genus. Otherwise each partition at each taxonomic level is colored uniquely. For the quantitative comparison of GORG-Tropics sample BATS248 was randomly subsampled to the median depth of the other 27 samples. Letters at each taxonomic rank refer to key marine archaea/bacterial groups (key left) and bar size is proportional to relative abundance. C) The proportion of phosphonate producers significantly increases with depth in the 28 GORG-Tropics samples. Each point is a sample (median SAGs $=241$ ). Producers are slightly more common in the Atlantic than the Pacific [Beta-binomial regression; Depth - Est $=0.0036, \mathrm{Err}=0.0014, \mathrm{t}=2.604, \mathrm{P}=0.02$; Ocean - Est $=-0.30$, $E r r=0.14, t=-2.08, P=0.05$; link=logit; $\log L=-78.179, d f=4$, resid $d f=24]$. $D)$ Phosphonate consumers are significantly more abundant in the Atlantic than the Pacific [Beta-binomial regression; Ocean - Est=-1.04, Err=0.27, $t=-3.81, P=7 e-4$; link=logit; $\log L=-80.425, d f=4$, resid $d f=24]$. Proportions [\%] are total producers or consumers divided by the total number GORG assemblies and are corrected using the estimated sequence recovery from assemblies (see methods). 
A

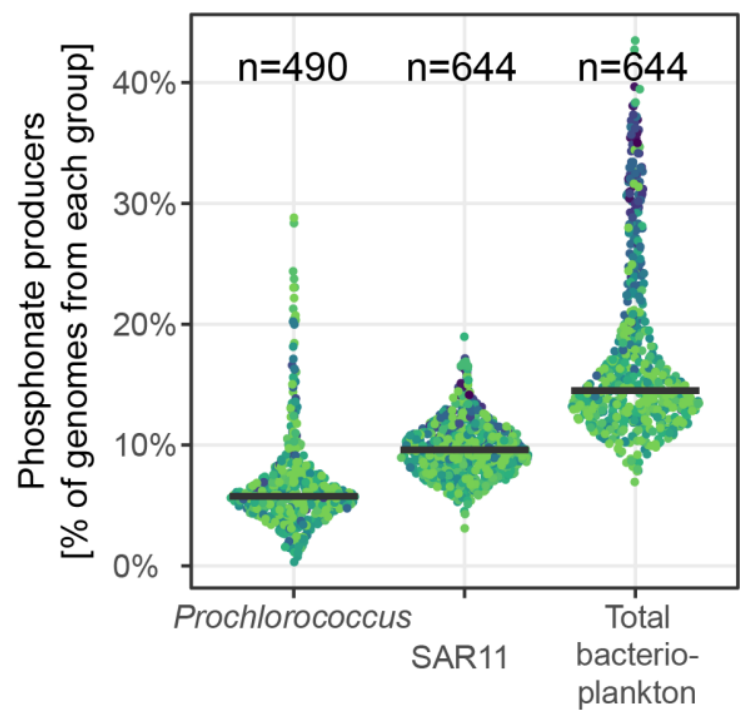

B

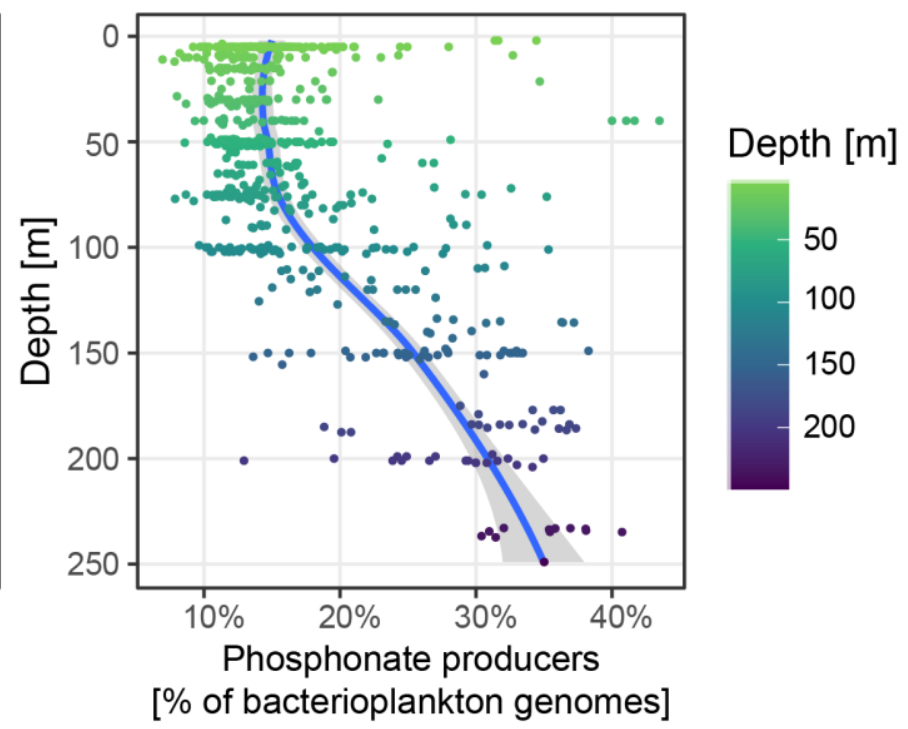

Figure 3: Phosphonate biosynthesis genes in surface ( $<300$ meters) ocean metagenomes

A) Phosphonate producers as the percentage of all bacterioplankton genomes estimated from combined BioGEOTRACES and Tara Oceans samples for Prochlorococcus, SAR11, and all Bacteria and Archaea combined. Black line is the median value for $n$ metagenome samples for each taxonomic group. B) The relationship between depth and percent phosphonate producers in the global ocean. The blue line is a simple Loess regression fit to the data. Norm. PepM is the normalized fraction genomes with PepM and is estimated as the length-normalized abundance of PepM divided by length-normalized abundance of taxon-specific marker genes. 
A

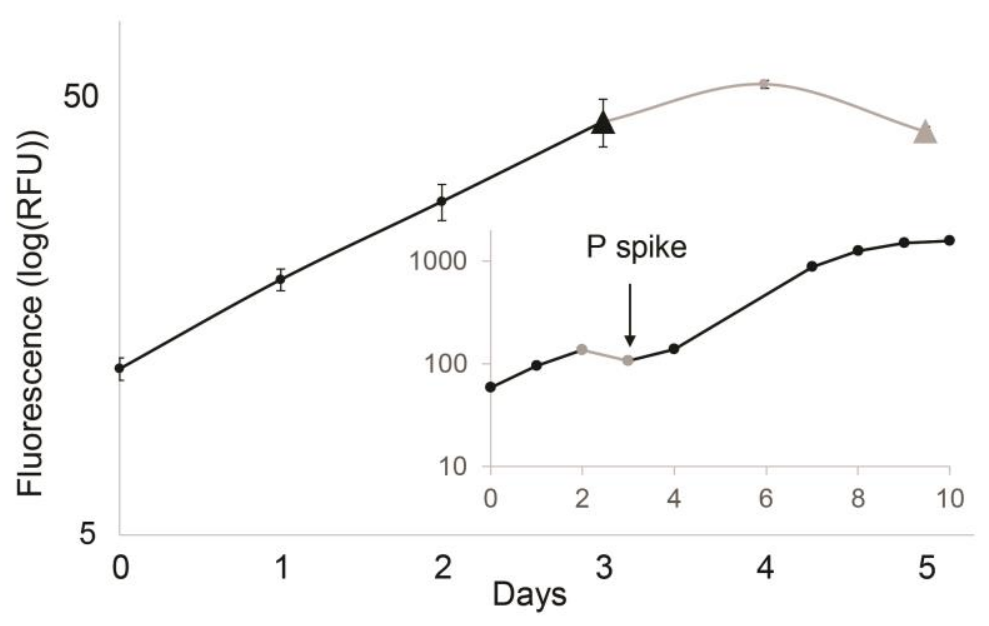

B

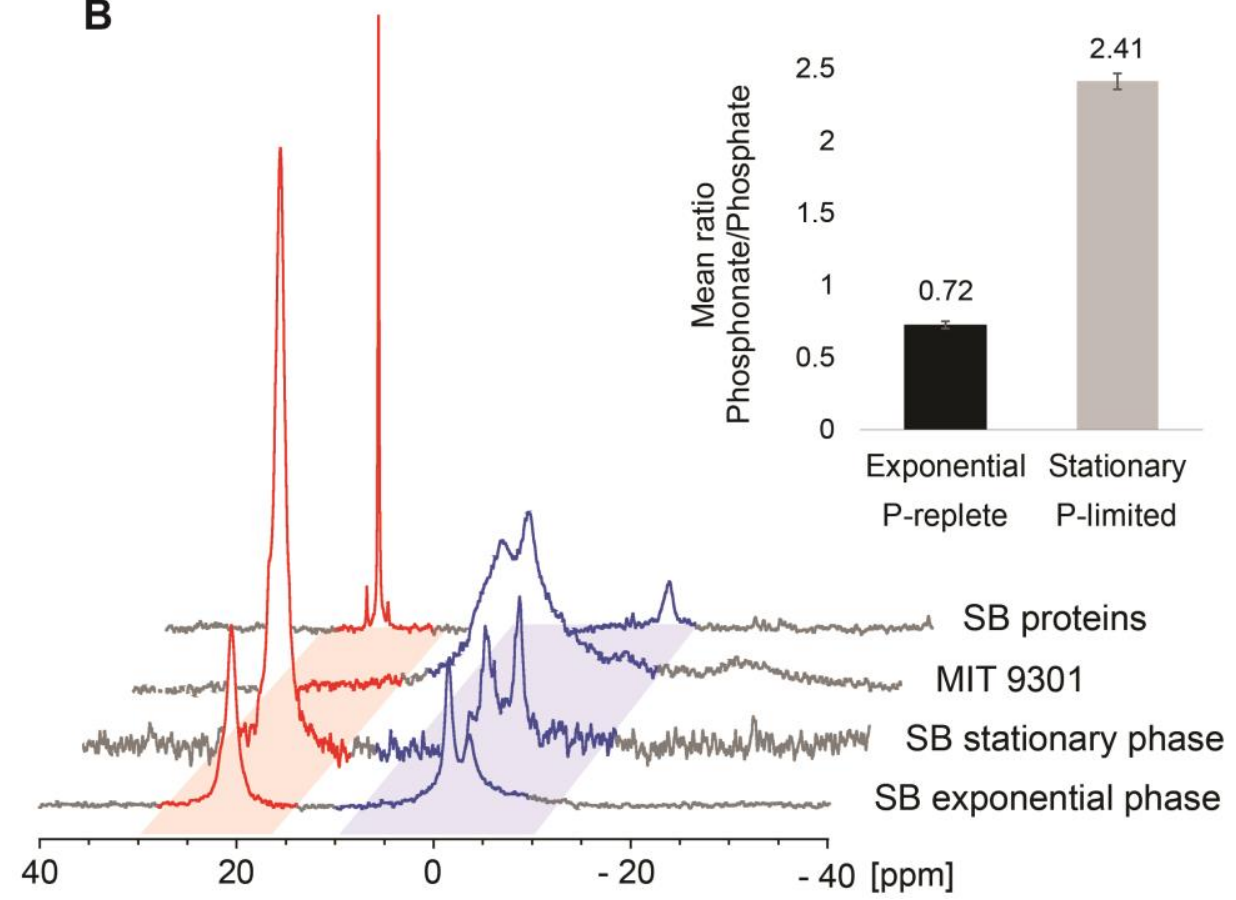

Figure 4: Phosphonate production by Prochlorococcus cultures

A) Prochlorococcus SB growth curve showing exponential phase (black) and stationary phase (grey) due to P-limitation. Triangle data points correspond to the days cultures were harvested in the different growth phases. Error bars are calculated based on the standard deviation between the biological duplicates. The inset represents the Prochlorococcus SB growth curve in medium with N/P = 350/1 in which inorganic phosphate was added on Day 3 to reach N/P $=16 / 1$. B) ${ }^{31} \mathrm{P}-\mathrm{NMR}$ spectra of Prochlorococcus SB whole cells harvested in exponential phase (P-replete) and in stationary phase (P-limited), the negative control Prochlorococcus MIT9301 harvested in exponential phase (P-replete) and the insoluble protein fraction of Prochlorococcus SB harvested in exponential growth phase. The phosphonate and phosphate regions of the spectra are indicated in red and blue respectively. While Prochlorococcus SB produces phosphonate and doubles its relative phosphonate content in P-limited stationary phase, the negative control, MIT9301 only produces phosphates. The histogram (inset) displays the mean Phosphonate/Phosphate ratios for Prochlorococcus SB cells harvested in exponential (black) and stationary (grey) phase calculated by integrating the phosphonate and phosphate peaks in Prochlorococcus SB whole cell ${ }^{31} \mathrm{P}-\mathrm{NMR}$ spectra obtained for the duplicates in each growth phase. Error bars correspond to the standard deviation of the biological replicate phosphonate/phosphate ratio values with $n=2$. 


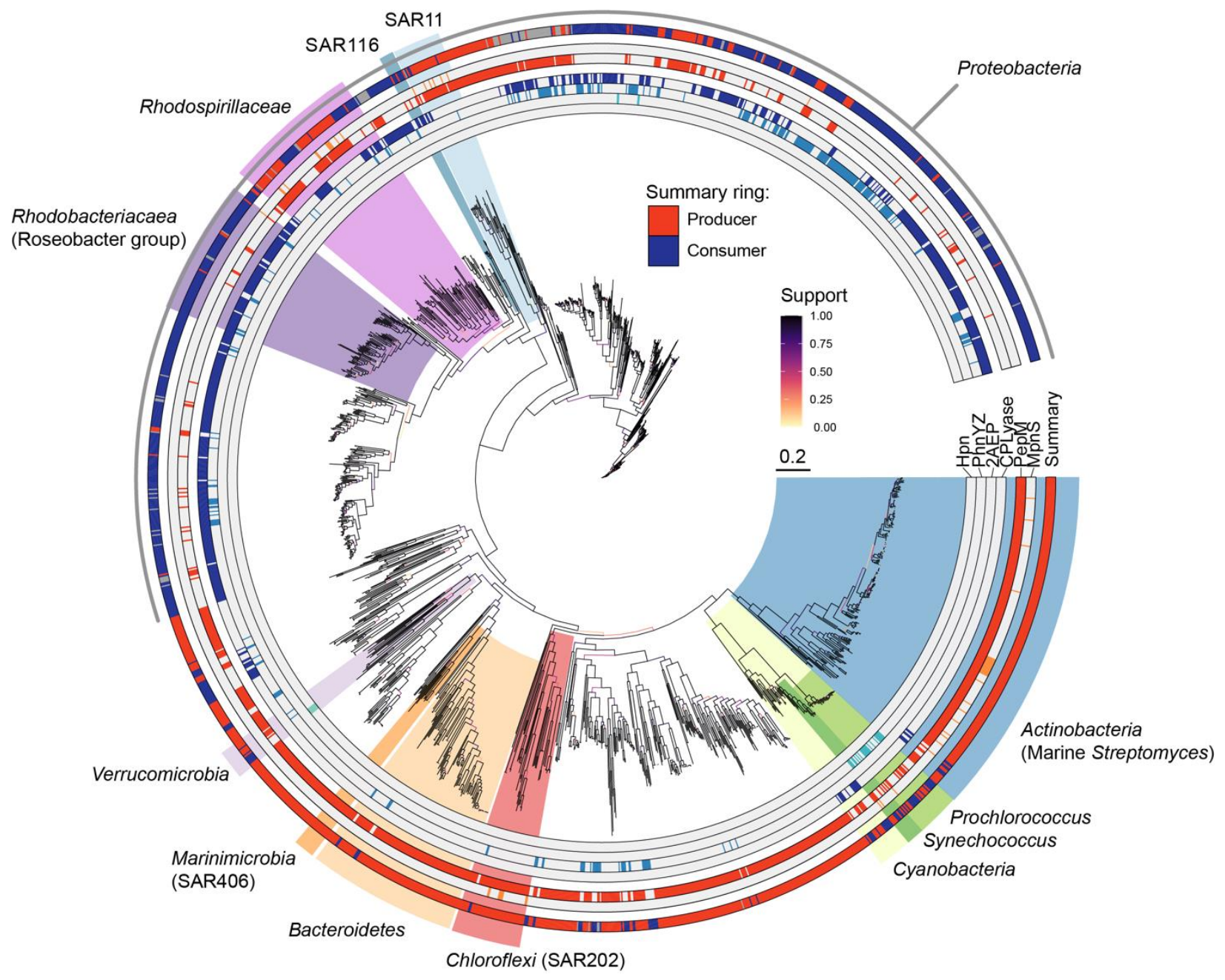

\section{Figure 5: Distribution of phosphonate biosynthesis and utilization pathways in bacterial genomes.}

Phylogeny is constructed from 120 concatenated, single-copy marker genes from 1890 bacterial genomes from MARMICRODB containing either a phosphonate catabolism or phosphonate biosynthesis pathway. Scale bar is 0.2 amino acid substitution and the tree is unrooted. Monophyletic taxonomic groups with marine representatives are highlighted. The presence of four different phosphonate catabolic pathways (Hpn: Phosphonate catabolism via HpnWXZ, PhnYZ: phosphite or methylphosphonate catabolism via PhnYZ4 ${ }^{4}$, AEP: 2-aminoethylphosphonate catabolism via phosphonoacetate, CPLyase: multisubunit C-P lyase system) is displayed in blue on an inner ring while phosphonate/methylphosphonate biosynthesis pathways (PepM and MpnS) are shown in red on the middle ring. The outer summary ring indicates whether a genome contains at least one catabolic pathway (blue), a phosphonate biosynthesis pathway (red), or both (dark grey, $<0.5 \%$ of all genomes). For simplification, the MpnS category also includes the functionally related enzyme HepDI64. 
A

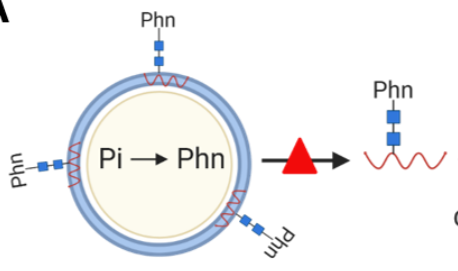

Phn producer

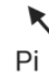

$\mathrm{Pi}$

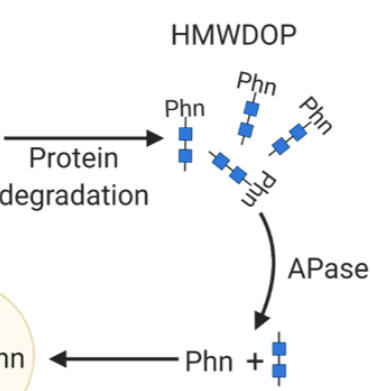

Phn consumer
B

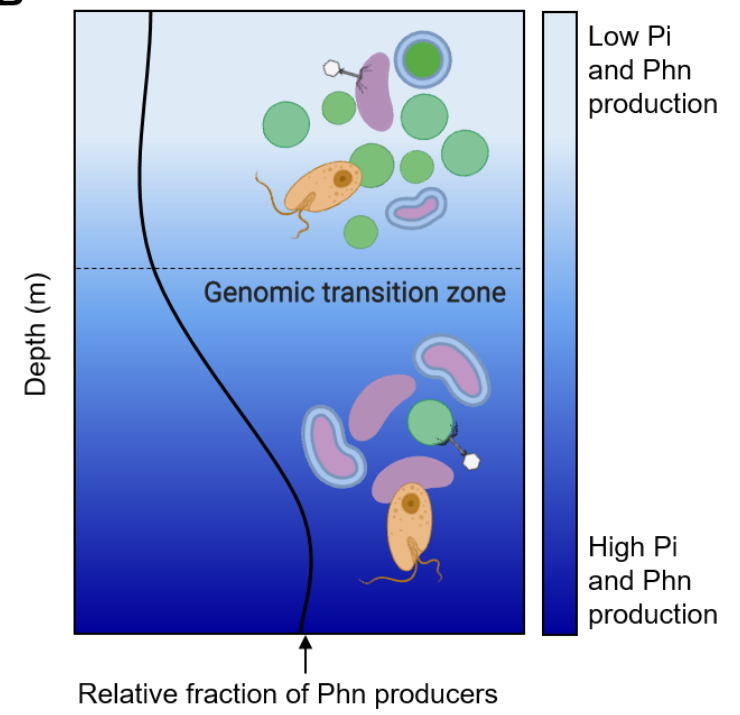

Figure 6: Microbial cycling of phosphonates in the upper ocean.

A) Microbes with PepM produce cell surface-layer phosphonoglycoproteins to reduce mortality from grazing or viral infection. Upon death of the cell phosphonoglycoproteins are released into seawater where heterotrophic microbes quickly remineralize proteins leaving phosphonoglycans to accumulate as high molecular weight dissolved organic phosphorus (HMWDOP). Phosphonates are hydrolyzed from glycans by alkaline phosphatases (APase) family enzymes and further hydrolyzed into inorganic phosphate $(\mathrm{Pi})$ by $\mathrm{C}-\mathrm{P}$ lyase or other hydrolytic pathways. Recycled Pi can then be used to produce new phosphonates. B) In the surface ocean, $\mathrm{Pi}$ is scarce and often limiting. Producing phosphonate as a mortality defense may be costly in terms of resource allocation. Therefore, the relative fraction of phosphonate producers in the microbial community is low. As nutrient availability increases through the genomic transition zone ${ }^{27}$ the benefit/cost ratio of phosphonate production increases and phosphonate producers are relatively more abundant. 


\section{References}

1. Karl, D. M. \& Björkman, K. M. Chapter 5 - Dynamics of Dissolved Organic Phosphorus. in Biogeochemistry of Marine Dissolved Organic Matter (Second Edition) (eds. Hansell, D. A. \& Carlson, C. A.) 233-334 (Academic Press, 2015).

2. Horsman, G. P. \& Zechel, D. L. Phosphonate Biochemistry. Chem. Rev. 117, 5704-5783 (2017).

3. Kolowith, L. C., Ingall, E. D. \& Benner, R. Composition and cycling of marine organic phosphorus. Limnol. Oceanogr. 46, 309-320 (2001).

4. Sosa, O. A., Casey, J. R. \& Karl, D. M. Methylphosphonate Oxidation in Prochlorococcus Strain MIT9301 Supports Phosphate Acquisition, Formate Excretion, and Carbon Assimilation into Purines. Appl. Environ. Microbiol. 85, (2019).

5. Repeta, D. J. et al. Marine methane paradox explained by bacterial degradation of dissolved organic matter. Nat. Geosci. 9, 884-887 (2016).

6. Martinez, A., Tyson, G. W. \& Delong, E. F. Widespread known and novel phosphonate utilization pathways in marine bacteria revealed by functional screening and metagenomic analyses. Environ. Microbiol. 12, 222-238 (2010).

7. Sosa, O. A., Repeta, D. J., DeLong, E. F., Ashkezari, M. D. \& Karl, D. M. Phosphate-limited ocean regions select for bacterial populations enriched in the carbon-phosphorus lyase pathway for phosphonate degradation. Environ. Microbiol. 21, 2402-2414 (2019).

8. Coleman, M. L. \& Chisholm, S. W. Ecosystem-specific selection pressures revealed through comparative population genomics. Proc. Natl. Acad. Sci. U. S. A. 107, 1863418639 (2010).

9. Dyhrman, S. T., Benitez-Nelson, C. R., Orchard, E. D., Haley, S. T. \& Pellechia, P. J. A microbial source of phosphonates in oligotrophic marine systems. Nat. Geosci. 2, 696-699 (2009). 
823

824

825

826

827

828

829

830

831

832

833

834

835

836

837

838

839

840

841

842

843

844

845

846

847

848

10. Metcalf, W. W. et al. Synthesis of methylphosphonic acid by marine microbes: a source for methane in the aerobic ocean. Science 337, 1104-1107 (2012).

11. Breitbarth, E., Oschlies, A. \& LaRoche, J. Physiological constraints on the global distribution of Trichodesmium? effect of temperature on diazotrophy. (2007).

12. Santoro, A. E., Richter, R. A. \& Dupont, C. L. Planktonic Marine Archaea. Ann. Rev. Mar. Sci. 11, 131-158 (2019).

13. Santoro, A. E. et al. Genomic and proteomic characterization of " Candidatus Nitrosopelagicus brevis": An ammonia-oxidizing archaeon from the open ocean. Proceedings of the National Academy of Sciences 112, 1173-1178 (2015).

14. $\mathrm{Yu}, \mathrm{X}$. et al. Diversity and abundance of phosphonate biosynthetic genes in nature. Proceedings of the National Academy of Sciences 110, 20759-20764 (2013).

15. Pachiadaki, M. G. et al. Charting the Complexity of the Marine Microbiome through SingleCell Genomics. Cell 179, 1623-1635.e11 (2019).

16. Berube, P. M. et al. Single cell genomes of Prochlorococcus, Synechococcus, and sympatric microbes from diverse marine environments. Sci Data 5, 180154 (2018).

17. Biller, S. J. et al. Marine microbial metagenomes sampled across space and time. Scientific Data 5, 180176 (2018).

18. Karsenti, E. et al. A holistic approach to marine eco-systems biology. PLoS Biol. 9, e1001177 (2011)

19. Morris, R. M. et al. SAR11 clade dominates ocean surface bacterioplankton communities. Nature 420, 806-810 (2002).

20. Biller, S. J., Berube, P. M., Lindell, D. \& Chisholm, S. W. Prochlorococcus: The structure and function of collective diversity. Nat. Rev. Microbiol. 13, 13-27 (2015).

21. Carini, P., White, A. E., Campbell, E. O. \& Giovannoni, S. J. Methane production by phosphate-starved SAR11 chemoheterotrophic marine bacteria. Nat. Commun. 5, 1-7 (2014). 
22. Schlitzer, R. et al. The GEOTRACES Intermediate Data Product 2017. Chem. Geol. 493, 210-223 (2018).

23. Karl, D. M. \& Lukas, R. The Hawaii Ocean Time-series (HOT) program: Background, rationale and field implementation. Deep Sea Res. Part 2 Top. Stud. Oceanogr. (1996).

24. Steinberg, D. K. et al. Overview of the US JGOFS Bermuda Atlantic Time-series Study (BATS): a decade-scale look at ocean biology and biogeochemistry. Deep Sea Res. Part 2 Top. Stud. Oceanogr. 48, 1405-1447 (2001).

25. Wright, M. N. \& Ziegler, A. ranger: A Fast Implementation of Random Forests for High Dimensional Data in C++ and R. arXiv [stat.ML] (2015).

26. Martin, B. D., Witten, D. \& Willis, A. D. Modeling microbial abundances and dysbiosis with beta-binomial regression. Ann. Appl. Stat. 14, 94-115 (2020).

27. Mende, D. R. et al. Environmental drivers of a microbial genomic transition zone in the ocean's interior. Nat Microbiol 2, 1367-1373 (2017).

28. Milici, M. et al. Diversity and community composition of particle-associated and free-living bacteria in mesopelagic and bathypelagic Southern Ocean water masses: Evidence of dispersal limitation in the Bransfield Strait: Bacteria in the deep Southern Ocean. Limnol. Oceanogr. 62, 1080-1095 (2017).

29. Nelson, C. E., Carlson, C. A., Ewart, C. S. \& Halewood, E. R. Community differentiation and population enrichment of Sargasso Sea bacterioplankton in the euphotic zone of a mesoscale mode-water eddy. Environ. Microbiol. 16, 871-887 (2014).

30. Barone, B. et al. The ecological and biogeochemical state of the North Pacific Subtropical Gyre is linked to sea surface height. J. Mar. Res. 77, 215-245 (2019).

31. Quin, L. D. \& Williams, A. J. Practical interpretation of P-31 Nmr Spectra and Computer Assisted Structure Verification. 132 (2004).

32. Metcalf, W. W. \& van der Donk, W. A. Biosynthesis of Phosphonic and Phosphinic Acid Natural Products. Annu. Rev. Biochem. 78, 65-94 (2009). 
875

876

877

33. Martin, P., Dyhrman, S. T., Lomas, M. W., Poulton, N. J. \& Van Mooy, B. A. S. Accumulation and enhanced cycling of polyphosphate by Sargasso Sea plankton in response to low phosphorus. Proc. Natl. Acad. Sci. U. S. A. 111, 8089-8094 (2014).

34. Temperton, B., Gilbert, J. A., Quinn, J. P. \& McGrath, J. W. Novel analysis of oceanic surface water metagenomes suggests importance of polyphosphate metabolism in oligotrophic environments. PLoS One 6, e16499 (2011).

35. Van Mooy, B. A. S., Rocap, G., Fredricks, H. F., Evans, C. T. \& Devol, A. H. Sulfolipids dramatically decrease phosphorus demand by picocyanobacteria in oligotrophic marine environments. Proc. Natl. Acad. Sci. U. S. A. 103, 8607-8612 (2006).

36. Becker, J. W., Hogle, S. L., Rosendo, K. \& Chisholm, S. W. Co-culture and biogeography of Prochlorococcus and SAR11. ISME J. 13, 1506-1519 (2019).

37. Coyne, M. J., Kalka-Moll, W., Tzianabos, A. O., Kasper, D. L. \& Comstock, L. E. Bacteroides fragilis NCTC9343 produces at least three distinct capsular polysaccharides: cloning, characterization, and reassignment of polysaccharide $\mathrm{B}$ and $\mathrm{C}$ biosynthesis loci. Infect. Immun. 68, 6176-6181 (2000).

38. Hilderbrand. The Role of Phosphonates in Living Systems. (Taylor \& Francis, 2018).

39. Wilkinson, B. J. \& Holmes, K. M. Staphylococcus aureus cell surface: capsule as a barrier to bacteriophage adsorption. Infect. Immun. 23, 549-552 (1979).

40. Decho, A. W. \& Gutierrez, T. Microbial Extracellular Polymeric Substances (EPSs) in Ocean Systems. Front. Microbiol. 8, 922 (2017).

41. Iwashkiw, J. A., Vozza, N. F., Kinsella, R. L. \& Feldman, M. F. Pour some sugar on it: the expanding world of bacterial protein O-linked glycosylation. Mol. Microbiol. 89, 14-28 (2013).

42. Lees-Miller, R. G. et al. A common pathway for O-linked protein-glycosylation and synthesis of capsule in A cinetobacter baumannii. Mol. Microbiol. 89, 816-830 (2013).

43. Faridmoayer, A., Fentabil, M. A., Mills, D. C., Klassen, J. S. \& Feldman, M. F. Functional 
characterization of bacterial oligosaccharyltransferases involved in O-linked protein glycosylation. J. Bacteriol. 189, 8088-8098 (2007).

44. Power, P. M., Seib, K. L. \& Jennings, M. P. Pilin glycosylation in Neisseria meningitidis occurs by a similar pathway to wzy-dependent O-antigen biosynthesis in Escherichia coli. Biochem. Biophys. Res. Commun. 347, 904-908 (2006).

45. Rice, K. et al. The predominance of nucleotidyl activation in bacterial phosphonate biosynthesis. Nat. Commun. 10, 3698 (2019).

46. White, A. K. \& Metcalf, W. W. Microbial Metabolism of Reduced Phosphorus Compounds. Annu. Rev. Microbiol. 61, 379-400 (2007).

47. Seed, K. D. Battling Phages: How Bacteria Defend against Viral Attack. PLoS Pathog. 11, e1004847 (2015).

48. Jürgens, K. \& Matz, C. Predation as a shaping force for the phenotypic and genotypic composition of planktonic bacteria. Antonie Van Leeuwenhoek 81, 413-434 (2002).

49. Partensky, F., Hess, W. R. \& Vaulot, D. Prochlorococcus, a marine photosynthetic prokaryote of global significance. Microbiol. Mol. Biol. Rev. 63, 106-127 (1999).

50. Fagan, R. P. \& Fairweather, N. F. Biogenesis and functions of bacterial S-layers. Nat. Rev. Microbiol. 12, 211-222 (2014).

51. Cordero, O. X. \& Polz, M. F. Explaining microbial genomic diversity in light of evolutionary ecology. Nat. Rev. Microbiol. 12, 263-273 (2014).

52. Nakamura, Y., Itoh, T., Matsuda, H. \& Gojobori, T. Biased biological functions of horizontally transferred genes in prokaryotic genomes. Nat. Genet. 36, 760-766 (2004).

53. Kettler, G. C. et al. Patterns and implications of gene gain and loss in the evolution of Prochlorococcus. PLoS Genet. 3, e231 (2007).

54. Coleman, M. L. et al. Genomic islands and the ecology and evolution of Prochlorococcus. Science 311, 1768-1770 (2006).

55. Frost, L. S., Leplae, R., Summers, A. O. \& Toussaint, A. Mobile genetic elements: the 
agents of open source evolution. Nat. Rev. Microbiol. 3, 722-732 (2005).

56. Van Mooy, B. A. S. et al. Phosphorus cycling. Major role of planktonic phosphate reduction in the marine phosphorus redox cycle. Science 348, 783-785 (2015).

57. Sannigrahi, P., Ingall, E. D. \& Benner, R. Nature and dynamics of phosphorus-containing components of marine dissolved and particulate organic matter. Geochim. Cosmochim. Acta 70, 5868-5882 (2006).

58. Granzow, B. N., Sosa, O. A., Gonnelli, M., Santinelli, C., Karl, D. M., \& Repeta, D. J. A sensitive fluorescent assay for measuring carbon-phosphorus lyase activity in aquatic systems. Limnol. Oceanogr. Methods (in revision).

59. Repeta, D. J. Chemical Characterization and Cycling of Dissolved Organic Matter. 21-63 (2014).

60. Haft, D. H. et al. TIGRFAMs and Genome Properties in 2013. Nucleic Acids Res. 41, D38795 (2013).

61. Ju, K.-S. et al. Discovery of phosphonic acid natural products by mining the genomes of 10,000 actinomycetes. Proc. Natl. Acad. Sci. U. S. A. 112, 12175-12180 (2015).

62. Cioni, J. P. et al. Cyanohydrin phosphonate natural product from Streptomyces regensis. J. Nat. Prod. 77, 243-249 (2014).

63. Chen, C. C. H. et al. Structure and kinetics of phosphonopyruvate hydrolase from Variovorax sp. Pal2: new insight into the divergence of catalysis within the PEP mutase/isocitrate lyase superfamily. Biochemistry 45, 11491-11504 (2006).

64. Born, D. A. et al. Structural basis for methylphosphonate biosynthesis. Science 358, 13361339 (2017).

65. Katoh, K. \& Standley, D. M. MAFFT multiple sequence alignment software version 7: improvements in performance and usability. Mol. Biol. Evol. 30, 772-780 (2013).

66. Ding, W., Baumdicker, F. \& Neher, R. A. panX: pan-genome analysis and exploration. Nucleic Acids Res. 46, e5 (2018). 
953

954

955

956

957

958

959

960

961

962

963

964

965

966

967

968

969

970

971

972

973

974

975

976

977

978

67. Milanese, A. et al. Microbial abundance, activity and population genomic profiling with mOTUs2. Nat. Commun. 10, 1014 (2019).

68. Steinegger, M. \& Söding, J. MMseqs2 enables sensitive protein sequence searching for the analysis of massive data sets. Nat. Biotechnol. 35, 1026-1028 (2017).

69. Capella-Gutiérrez, S., Silla-Martínez, J. M. \& Gabaldón, T. trimAl: a tool for automated alignment trimming in large-scale phylogenetic analyses. Bioinformatics 25, 1972-1973 (2009).

70. Chaumeil, P.-A., Mussig, A. J., Hugenholtz, P. \& Parks, D. H. GTDB-Tk: a toolkit to classify genomes with the Genome Taxonomy Database. Bioinformatics (2019) doi:10.1093/bioinformatics/btz848.

71. Parks, D. H. et al. A standardized bacterial taxonomy based on genome phylogeny substantially revises the tree of life. Nat. Biotechnol. 36, 996-1004 (2018).

72. Price, M. N., Dehal, P. S. \& Arkin, A. P. FastTree 2--approximately maximum-likelihood trees for large alignments. PLoS One 5, e9490 (2010).

73. Whelan, S. \& Goldman, N. A general empirical model of protein evolution derived from multiple protein families using a maximum-likelihood approach. Mol. Biol. Evol. 18, 691-699 (2001).

74. Yu, G., Smith, D. K., Zhu, H., Guan, Y. \& Lam, T. T. ggtree : an r package for visualization and annotation of phylogenetic trees with their covariates and other associated data. Methods Ecol. Evol. 8, 28-36 (2017).

75. Huerta-Cepas, J., Serra, F. \& Bork, P. ETE 3: Reconstruction, Analysis, and Visualization of Phylogenomic Data. Mol. Biol. Evol. 33, 1635-1638 (2016).

76. Robinson, D. F. \& Foulds, L. R. Comparison of phylogenetic trees. Math. Biosci. 53, 131$147(1981)$.

77. Huerta-Cepas, J. et al. eggNOG 4.5: a hierarchical orthology framework with improved functional annotations for eukaryotic, prokaryotic and viral sequences. Nucleic Acids Res. 
44, D286-93 (2016).

980

981

982

983

984

985

986

987

988

989

990

991

992

993

994

995

996

997

998

999

1000

1001

1002

1003

1004

78. Huerta-Cepas, J. et al. Fast Genome-Wide Functional Annotation through Orthology Assignment by eggNOG-Mapper. Mol. Biol. Evol. 34, 2115-2122 (2017).

79. Kanehisa, M. \& Goto, S. KEGG: kyoto encyclopedia of genes and genomes. Nucleic Acids Res. 28, 27-30 (2000).

80. Rivals, I., Personnaz, L., Taing, L. \& Potier, M.-C. Enrichment or depletion of a GO category within a class of genes: which test? Bioinformatics 23, 401-407 (2006).

81. Yu, G., Wang, L.-G., Han, Y. \& He, Q.-Y. clusterProfiler: an R Package for Comparing Biological Themes Among Gene Clusters. OMICS 16, 284-287 (2012).

82. Avrani, S., Wurtzel, O., Sharon, I., Sorek, R. \& Lindell, D. Genomic island variability facilitates Prochlorococcus--virus coexistence. Nature 474, 604-608 (2011).

83. Dufresne, A. et al. Unraveling the genomic mosaic of a ubiquitous genus of marine cyanobacteria. Genome Biol. 9, R90 (2008).

84. Parks, D. H., Imelfort, M., Skennerton, C. T., Hugenholtz, P. \& Tyson, G. W. CheckM: assessing the quality of microbial genomes recovered from isolates, single cells, and metagenomes. Genome Res. 25, 1043-1055 (2015).

85. Buchfink, B., Xie, C. \& Huson, D. H. Fast and sensitive protein alignment using DIAMOND. Nat. Methods 12, 59-60 (2015).

86. Salt, L. A., Heuven, S., Claus, M. E., Jones, E. M. \& Baar, H. J. W. Rapid acidification of mode and intermediate waters in the southwestern Atlantic Ocean. (2015).

87. Rijkenberg, M. J. A. et al. The distribution of dissolved iron in the West Atlantic Ocean. PLoS One 9, e101323 (2014).

88. Wyatt, N. J. et al. Biogeochemical cycling of dissolved zinc along the GEOTRACES South Atlantic transect GA10 at 40 S. Global Biogeochem. Cycles 28, 44-56 (2014).

89. Speich, S. et al. Environmental context of all samples from the Tara Oceans Expedition (2009-2013), about the water column features at the sampling location. in (PANGAEA, 
2017).

1006

1007

1008

1009

1010

1011

1012

1013

1014

1015

1016

1017

1018

1019

1020

1021

1022

1023

1024

1025

1026

1027

1028

1029

1030

90. Weinhold, L. et al. A Random Forest Approach for Bounded Outcome Variables. null 1-20 (2019).

91. Teschendorff, A. E. Avoiding common pitfalls in machine learning omic data science. Nat. Mater. 18, 422-427 (2019).

92. Zuber, V. \& Strimmer, K. High-Dimensional Regression and Variable Selection Using CAR Scores. Stat. Appl. Genet. Mol. Biol. 10, (2011).

93. Kursa, M. B., Rudnicki, W. R. \& Others. Feature selection with the Boruta package. J. Stat. Softw. 36, 1-13 (2010).

94. Wood, S. N. Generalized Additive Models: An Introduction with R, Second Edition. (CRC Press, 2017).

95. Moore, L. R. et al. Culturing the marine cyanobacterium Prochlorococcus: Prochlorococcus culturing. Limnol. Oceanogr. Methods 5, 353-362 (2007).

96. Morris, J. J., Kirkegaard, R., Szul, M. J., Johnson, Z. I. \& Zinser, E. R. Facilitation of robust growth of Prochlorococcus colonies and dilute liquid cultures by "helper" heterotrophic bacteria. Appl. Environ. Microbiol. 74, 4530-4534 (2008).

97. Saito, M. A., Moffett, J. W., Chisholm, S. W. \& Waterbury, J. B. Cobalt limitation and uptake in Prochlorococcus. Limnol. Oceanogr. 47, 1629-1636 (2002).

98. Waterbury, J. B. \& Willey, J. M. [6] Isolation and growth of marine planktonic cyanobacteria. in Methods in Enzymology vol. 167 100-105 (Academic Press, 1988).

99. Zinser, E. R. et al. Prochlorococcus ecotype abundances in the North Atlantic Ocean as revealed by an improved quantitative PCR method. Appl. Environ. Microbiol. 72, 723-732 (2006).

100. Malmstrom, R. R. et al. Temporal dynamics of Prochlorococcus ecotypes in the Atlantic and Pacific oceans. ISME J. 4, 1252-1264 (2010).

101.Strickland, J. D. H. \& Parsons, T. R. A practical handbook of seawater analysis. (Fisheries 
1031 Research Board of Canada, 1972).

1032 102.Murphy, J. \& Riley, J. P. A modified single solution method for the determination of

1033 phosphate in natural waters. Anal. Chim. Acta 27, 31-36 (1962).

1034 103.Karl, D. M., Winn, C. D. \& Wong, D. C. L. RNA synthesis as a measure of microbial growth

1035 in aquatic environments. II. Field applications. Mar. Biol. 64, 13-21 (1981).

1036 104. Hutchins, D. A. et al. Irreversibly increased nitrogen fixation in Trichodesmium

1037 experimentally adapted to elevated carbon dioxide. Nat. Commun. 6, 8155 (2015).

1038 105. Menzel, P., Ng, K. L. \& Krogh, A. Fast and sensitive taxonomic classification for

1039 metagenomics with Kaiju. Nat. Commun. 7, 11257 (2016).

1040 106.Lahiri, S. D., Zhang, G., Dunaway-Mariano, D. \& Allen, K. N. Diversification of function in 1041 the haloacid dehalogenase enzyme superfamily: The role of the cap domain in hydrolytic 1042 phosphoruscarbon bond cleavage. Bioorg. Chem. 34, 394-409 (2006). 